\title{
UNMATING OF RATIONAL MAPS, SUFFICIENT CRITERIA AND EXAMPLES
}

\author{
DANIEL MEYER
}

\begin{abstract}
Douady and Hubbard introduced the operation of mating of polynomials. This identifies two filled Julia sets and the dynamics on them via external rays. In many cases one obtains a rational map. Here the opposite question is tackled. Namely we ask when a given (postcritically finite) rational map $f$ arises as a mating. A sufficient condition when this is possible is given. If this condition is satisfied, we present a simple explicit algorithm to unmate the rational map. This means we decompose $f$ into polynomials, that when mated yield $f$. Several examples of unmatings are presented.
\end{abstract}

\section{INTRODUCTION}

Douady and Hubbard observed that often "one can find" Julia sets of polynomials within Julia sets of rational maps. This prompted them to introduce the operation of mating of polynomials, see Dou83. It is a way to glue together two (connected and locally connected) filled Julia sets along their boundaries. The dynamics then descends to the quotient. Somewhat surprisingly one often obtains a map that is topologically conjugate to a rational map.

Thurston's celebrated theorem on the classification of rational maps among (postcritically finite) topological rational maps (see [DH93]) was largely inspired to give a complete answer when the mating of two polynomials results in (i.e., is topologically conjugate to) a rational map.

Here the opposite question is considered. Namely we ask whether a given rational map $f$ is a mating. This means to decide whether $f$ arises as (i.e., is topologically conjugate to) a mating of polynomials $P_{\mathrm{w}}, P_{\mathrm{b}}$. If the answer is yes, one wishes to unmate the map $f$, i.e., obtain the polynomials $P_{\mathrm{w}}, P_{\mathrm{b}}$.

The author has recently shown that in the case when $f$ is postcritically finite and the Julia set of $f$ is the whole Riemann sphere, that every sufficiently high iterate indeed arises as a mating, see [Mey11] and $\mathrm{Mey}$. Indeed the result remains true for expanding Thurston maps.

The purpose of this paper is twofold. The first is to explain the methods used in Mey11 and Mey. Namely we present a sufficient condition that $f$ arises as a mating of polynomials $P_{\mathrm{w}}, P_{\mathrm{b}}$. A simple algorithm to unmate $f$ is presented, i.e., to obtain $P_{\mathrm{w}}, P_{\mathrm{b}}$ in a simple combinatorial fashion. The presentation is largely expository, we do not attempt to provide full proofs (which can be found in Mey11 and $\mathrm{Mey}]$ and are somewhat technical).

The second purpose is to contrast the two opposite cases of rational (postcritically finite) rational maps. Namely we contrast hyperbolic maps, where each critical

Date: July 1, 2018. 
point is contained in the Fatou set, with maps where each critical point is contained in the Julia set (equivalently the Julia set is the whole Riemann sphere $\widehat{\mathbb{C}}$ ).

The following contrasting theorems are obtained.

Theorem 4.8. Let $f: \widehat{\mathbb{C}} \rightarrow \widehat{\mathbb{C}}$ be a hyperbolic, postcritically finite, rational map, with \# $\operatorname{post}(f)=3$, that is not a polynomial. Then $f$ does not arise as a mating.

Theorem 7.3, Let $f: \widehat{\mathbb{C}} \rightarrow \widehat{\mathbb{C}}$ be a postcritically finite rational map, with Julia set $\mathcal{J}(f)=\widehat{\mathbb{C}}$ and $\# \operatorname{post}(f)=3$. Then $f$ or $f^{2}$ arises as a mating.

In the case when the rational map $f$ is hyperbolic (and postcritically finite) there is a necessary and sufficient condition that $f$ arises as a mating. Namely $f$ arises as a mating if and only if $f$ has an equator $\mathcal{E}$. This is a Jordan curve $\mathcal{E} \subset \widehat{\mathbb{C}} \backslash \operatorname{post}(f)$ such that $f^{-1}(\mathcal{E})$ is orientation-preserving isotopic to $\mathcal{E}$ rel. post $(f)$. This theorem seems to be folklore, but does not appear (to the knowledge of the author) in the literature. We give a proof here for the convenience of the reader, see Theorem 4.2

The existence of an equator however is not the right condition for $f$ to arise as a mating in the non-hyperbolic case. There are many examples of maps, in particularly Lattès maps and maps where \# post $(f)=3$, which can be shown to have no equator (Proposition 4.3, Proposition 4.4 and Corollary 4.5). Nevertheless, it is known that many of these maps do indeed arise as a mating.

A sufficient condition that a postcritically finite rational map $f$ with Julia set $\mathcal{J}(f)=\widehat{\mathbb{C}}$ arises as a mating is the existence of a pseudo-equator. This means there is a Jordan curve $\mathcal{C} \subset \widehat{\mathbb{C}}$ that contains all postcritical points. Furthermore there is a pseudo-isotopy $H$ rel. post $(f)$ that deforms $\mathcal{C}$ to $\mathcal{C}^{1}:=f^{-1}(\mathcal{C})$ in an elementary, orientation-preserving way. Such a pseudo-equator is not only sufficient for $f$ to arise as a mating, but allows one to unmate $f$ as well. More precisely from the pseudo-equator (i.e., from the way $\mathcal{C}$ is deformed to $\mathcal{C}^{1}$ ) one obtains a matrix. From this matrix one can obtain the critical portraits of two polynomials $P_{\mathrm{w}}, P_{\mathrm{b}}$. These critical portraits determine $P_{\mathrm{w}}, P_{\mathrm{b}}$ uniquely. The map $f$ is (topologically conjugate to) the topological mating of $P_{\mathrm{w}}, P_{\mathrm{b}}$.

The question arises whether our sufficient condition is in fact necessary. The answer however turns out to be no as an explicit example shows.

The organization of this paper is as follows. In Section 2 we review Moore's theorem. While this theorem is essential in the theory of matings, one usually only encounters a weak form of the theorem. We present a stronger version that deserves to be much better known.

In Section 3 we review the mating construction, as well as relevant theorems.

In Section 4 we consider hyperbolic rational maps. We consider equators, existence and their connection to matings.

Section 5 introduces an example (it is a Lattès map), which is used in the following as an illustration.

The sufficient criterion for $f$ to arise as a mating mating from Mey11 and Mey for (postcritically finite) rational maps (whose Julia set is the whole sphere) is presented in Section 6. Namely $f$ arises as a mating if $f$ has a pseudo-equator.

An equivalent formulation of the sufficient criterion is given in Section 7 Let $\mathcal{C} \subset \widehat{\mathbb{C}}$ be a Jordan curve with post $(f) \subset \mathcal{C}$ we consider the preimages of the two components of $\widehat{\mathbb{C}} \backslash \mathcal{C}$. We color one component of $\widehat{\mathbb{C}} \backslash \mathcal{C}$ white, the other black. Each component of $\widehat{\mathbb{C}} \backslash f^{-1}(\mathcal{C})$ is a preimage of one component of $\widehat{\mathbb{C}} \backslash \mathcal{C}$. The 
closure of one such component is called a 1-tile. It it colored white/black if is mapped by $f$ to the white/black component of $\widehat{\mathbb{C}} \backslash \mathcal{C}$ respectively. The 1-tiles tile the sphere in a checkerboard fashion. At critical points several 1-tiles intersect. At each critical point we assign a connection. This is a formal assignment which 1-tiles are "connected" at this critical point.

The existence of a pseudo-equator is equivalent to the existence of connections at each critical point, such that the white 1-tiles form a spanning tree. Furthermore the "outline" of this spanning tree must be orientation-preserving isotopic to the Jordan curve $\mathcal{C}$.

We will need a description of polynomials that is adapted to matings, i.e., a description in terms of external angles. The one that best suits our needs is the description via critical portraits, introduced by Bielefeld-Fisher-Hubbard and Poirier (see BFH92 and Poi09]). Essentially one records the external angles at critical points. Each such critical portrait yields a (unique up to affine conjugation) monic polynomial. We review the necessary material in Section 8

In Section 9 we present the algorithm to unmate rational maps. Namely one obtains from a pseudo-equator a matrix that encodes how one edge in $\mathcal{C}$ is deformed to several in $\mathcal{C}^{1}:=f^{-1}(\mathcal{C})$ by the corresponding pseudo-isotopy. From the PerronFrobenius eigenvector one obtains the critical portraits of the two polynomials into which $f$ unmates.

In Section 10 we present several examples of unmatings of rational maps.

In Section 11 we show that the existence of a pseudo-equator is not necessary for a rational map $f$ to arise as a mating. Namely we construct a (rational, postcritically finite) rational map $f$, whose Julia set is the whole sphere. In fact $f$ is constructed as the (topological) mating of two (quadratic postcritically finite) polynomials. We then show that $f$ has no pseudo-equator.

We end the paper in Section 12 with several open questions.

1.1. Notation. The Riemann sphere is denoted by $\widehat{\mathbb{C}}=\mathbb{C} \cup\{\infty\}$, the 2 -sphere by $S^{2}$ (which is a topological object, not equipped with a conformal structure). The unit circle is $S^{1}$, which will often be identified with $\mathbb{R} / \mathbb{Z}$. The circle at infinity is $\left\{\infty \cdot e^{2 \pi i \theta} \mid \theta \in \mathbb{R} / \mathbb{Z}\right\}$, the extension of $\mathbb{C}$ to the circle at infinity is $\overline{\mathbb{C}}=\mathbb{C} \cup\left\{\infty \cdot e^{2 \pi i \theta} \mid\right.$ $\theta \in \mathbb{R} / \mathbb{Z}\}$. The Julia set is $\mathcal{J}$, the filled Julia set $\mathcal{K}$.

We will often color objects (polynomials, sets) black and white. White objects will usually be equipped with a subscript $\mathrm{w}$, black objects will be equipped with a subscript b.

If $f=a_{0}+a_{n}(z-c)^{n}+\ldots$, then the local degree of $f$ at $c$ is $\operatorname{deg}_{f}(c)=n$.

\section{MoOre's THEOREM}

Let $X$ be a compact metric space. An equivalence relation $\sim$ on $X$ is called closed if $\{(x, y) \in X \times X \mid x \sim y\}$ is a closed subset of $X \times X$. Equivalently, for any two convergent sequences $\left(x_{n}\right),\left(y_{n}\right)$ in $X$ with $x_{n} \sim y_{n}$ for all $n \in \mathbb{N}$, it holds $\lim x_{n}=\lim y_{n}$.

A map $f: X \rightarrow Y$ induces an equivalence relation on $X$ as follows. For all $x, x^{\prime} \in X$ let $x \sim x^{\prime}$ if and only if $f(x)=f\left(x^{\prime}\right)$.

Lemma 2.1. Let $X, Y$ be compact metric spaces, and $f: X \rightarrow Y$ be a continuous surjection. Let $\sim$ be the equivalence relation on $X$ induced by $f$. Then $\sim$ is closed and $X / \sim$ is homeomorphic to $Y$. 
Proof. Let $x_{n} \rightarrow x$ and $y_{n} \rightarrow y$ be two convergent sequence in $X$, such that $x_{n} \sim y_{n}$ for all $n \in \mathbb{N}$. Then

$$
f(x)=\lim f\left(x_{n}\right)=\lim f\left(y_{n}\right)=f(y) .
$$

Thus $x \sim y$. This shows that $\sim$ is closed.

Define $h: X / \sim \rightarrow Y$ by $h([x])=f(x)$. Clearly this is a well-defined bijective map. Let $\pi: X \rightarrow X / \sim$ be the quotient map. Consider an open set $V \subset Y$. Then $f^{-1}(V)=\pi^{-1}\left(h^{-1}(V)\right)$ is open. Thus $h^{-1}(V)$ is open in $X / \sim$, by the definition of the quotient topology. Thus $h$ is continuous, thus a homeomorphism.

Moore's theorem is of central importance in the theory of matings. However in the literature on matings one usually only encounters a weak form of the theorem.

Definition 2.2. An equivalence relation $\sim$ on $S^{2}$ is called of Moore-type if

(1) $\sim$ is not trivial, i.e., there are at least two distinct equivalence classes;

(2) $\sim$ is closed;

(3) each equivalence class $[x]$ is connected;

(4) no equivalence class separates $S^{2}$, i.e., $S^{2} \backslash[x]$ is connected for each equivalence class $[x]$.

The reason for the name "Moore-type" is the classical theorem of Moore that asserts that if $\sim$ is of Moore-type, then the quotient space $S^{2} / \sim$ is homeomorphic to $S^{2}$. This statement is however true in a stronger form, which we present next.

Definition 2.3. A homotopy $H: X \times[0,1] \rightarrow X$ is called a pseudo-isotopy if $H: X \times[0,1) \rightarrow X$ is an isotopy (i.e., $H(\cdot, t)$ is a homeomorphism for all $t \in[0,1)$ ). We will always assume that $H(x, 0)=x$ for all $x \in X$.

Given a set $A \subset S^{2}$, we call $H$ a pseudo-isotopy rel. $A$ if $H$ is a homotopy rel. $A$, i.e., if $H(a, t)=a$ for all $a \in A, t \in[0,1]$. We call the map $h:=H(\cdot, 1)$ the end of the pseudo-isotopy $H$.

We interchangeably write $H(\cdot, t)=H_{t}(\cdot)$ to unclutter notation.

Lemma 2.4. Let $H: S^{2} \times I \rightarrow S^{2}$ be a pseudo-isotopy. We consider the equivalence relation induced by the end of the pseudo-isotopy, i.e., for $x, y \in S^{2}$

$$
x \sim y \quad \Leftrightarrow \quad H_{1}(x)=H_{1}(y) .
$$

Then $\sim$ is of Moore-type.

Proof. Since $S^{2}$ is not contractable, it follows that $H_{1}$ is surjective. Thus $\sim$ is not trivial, i.e., not all points in $S^{2}$ are equivalent. Lemma 2.1 shows that $\sim$ is closed. Furthermore $S^{2} / \sim=S^{2}$.

Consider an equivalence class $[x]$. Assume it is not connected. Then there is a Jordan curve $C$ that separates $[x]$. Let $x_{0} \in[x]$. We can assume that $H$ keeps $x_{0}$ fixed, otherwise we can compose $H$ with an isotopy such that the composition keeps $x_{0}$ fixed. There is a $\delta>0$ such that $\operatorname{dist}\left(H_{t}(C), x_{0}\right)>\delta$ for all $t \in[0,1]$.

We call the component of $S^{2} \backslash C$ containing $x_{0}$ the interior of $C$, the other one the exterior of $C$. Consider a point $y_{0} \in[x]$ in the exterior of $C$. Let $y_{t}:=H_{t}\left(y_{0}\right)$ for $t \in[0,1]$. By assumption $\lim _{t \rightarrow 1} y_{t}=x_{0}$. Thus there is a $t_{0} \in[0,1)$ such that $\left|y_{t_{0}}-x_{0}\right|<\delta / 2$. It follows that $y_{t_{0}}$ is in the same component of $C_{t_{0}}:=H_{t_{0}}(C)$ as $x_{0}$. This is impossible. 
Assume now that there is an equivalence class $[x]$ that separates $S^{2}$, i.e., $S^{2} \backslash[x]$ has (at least) two components. We already know that each distinct equivalence class $[y]$ is connected. Thus it follows that each equivalence class $[y] \neq[x]$ is contained in one of the components of $S^{2} \backslash[x]$. It follows that if we remove the point $[x]$ from $S^{2} / \sim$ we obtain a disconnected set. This violates the fact that $S^{2} / \sim$ is homeomorphic to $S^{2}$.

Theorem 2.5 (Moore, 1925). Let $\sim$ be an equivalence relation on $S^{2}$. Then $\sim$ is of Moore-type if and only if $\sim$ can be realized as the end of a pseudo-isotopy $H: S^{2} \times[0,1] \rightarrow S^{2}$ (i.e., $x \sim y \Leftrightarrow H_{1}(x)=H_{1}(y)$ ).

The "if-direction" was shown in Lemma 2.4. A proof of the other direction can be found in Dav86, Theorem 25.1 and Theorem 13.4]. From Lemma 2.1 we immediately recover the original form of Moore's theorem, i.e., that $S^{2} / \sim$ is homeomorphic to $S^{2}$.

The following theorem was originally proved by Baer, see Bae27] and Bae28, a more modern treatment can be found in Eps66, Theorem 2.1].

Theorem 2.6. Let $P \subset S^{2}$ be a finite set and $\mathcal{C}, \mathcal{C}^{\prime}: S^{1} \rightarrow S^{2} \backslash P$ be Jordan curves that are homotopic in $S^{2} \backslash P$, i.e., there is a homotopy $H: S^{1} \times I \rightarrow S^{2} \backslash P$ such that $H\left(S^{1}, 0\right)=\mathcal{C}$ and $H\left(S^{1}, 1\right)=\mathcal{C}^{\prime}$. Furthermore each component of $S^{2} \backslash \mathcal{C}$ contains at least a point in $P$.

Then $\mathcal{C}, \mathcal{C}^{\prime}$ are isotopic rel. $P$, i.e., there is an isotopy $K: S^{2} \times I \rightarrow S^{2}$ rel. $P$ such that $K(\cdot, 0)=\operatorname{id}_{S^{2}}$ and $K(\mathcal{C}, 1)=\mathcal{C}^{\prime}$.

\section{Mating of POLYNOMials}

An excellent introduction to matings can be found in Mil04. In fact this paper was the main inspiration for the papers [Mey11 and Mey. See also [Wit88] and SL00].

We still give the basic definitions here, but we will be brief.

Recall that a Thurston map is an orientation-preserving branched covering map $f: S^{2} \rightarrow S^{2}$ of degree at least 2, that is postcritically finite (see BM11] for more background). A Thurston map $f$ is called a Thurston polynomial if there is a totally invariant point, i.e., a point $\infty \in S^{2}$ such that $f(\infty)=f^{-1}(\infty)=\infty$.

3.1. Topological mating. Let $P$ be a monic polynomial (i.e., the coefficient of the leading term is 1 ) with connected and locally connected filled Julia set $\mathcal{K}$. Let $\phi: \widehat{\mathbb{C}} \backslash \overline{\mathbb{D}} \rightarrow \widehat{\mathbb{C}} \backslash \mathcal{K}$ be the Riemann map normalized by $\phi(\infty)=\infty$ and $\phi^{\prime}(\infty)=$ $\lim _{z \rightarrow \infty} \phi(z) / z>0$ (in fact then $\phi^{\prime}(\infty)=1$ ). By Carathéodory's theorem (see for example [Mil99, Theorem 17.14]) $\phi$ extends continuously to

$$
\sigma: S^{1}=\partial \overline{\mathbb{D}} \rightarrow \partial \mathcal{K}=\mathcal{J},
$$

where $\mathcal{J}$ is the Julia set of $P$. We call the map $\sigma$ the Carathédory semi-conjugacy of $\mathcal{J}$. We remind the reader that every postcritically finite polynomial has connected and locally connected filled Julia set (see for example Mil99, Theorem 19.7]).

Consider the equivalence relation on $S^{1}$ induced by the Carathéodory semiconjugacy, namely

$$
s \sim t: \Leftrightarrow \sigma(s)=\sigma(t),
$$

for all $s, t \in S^{1}$. Lemma 2.1 yields that $S^{1} / \sim$ is homeomorphic to $\mathcal{J}$, where the homeomorphism is given by $h: S^{1} / \sim \rightarrow \mathcal{J},[s] \mapsto \sigma(s)$. Böttcher's theorem (see for 
example [Mil99, $\S 9])$ says that the Riemann map $\phi$ conjugates $z^{d}$ to the polynomial $P$ on $\widehat{\mathbb{C}} \backslash \overline{\mathbb{D}}$, where $d=\operatorname{deg} P$. This means that the following diagram commutes,

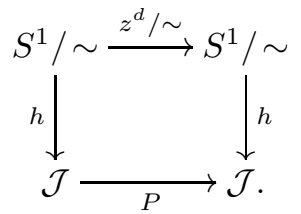

It will be convenient to identify the unit circle $S^{1}$ with $\mathbb{R} / \mathbb{Z}$. We still write

$$
\sigma: \mathbb{R} / \mathbb{Z} \rightarrow \mathcal{J}=\partial \mathcal{K}
$$

for the Carathéodory semi-conjugacy.

Consider two monic polynomials $P_{\mathrm{w}}, P_{\mathrm{b}}$ (called the white and the black polynomial) of the same degree with connected and locally connected Julia sets. Let $\sigma_{\mathrm{w}}, \sigma_{\mathrm{b}}$ be the Carathéodory semi-conjugacies of their Julia sets $\mathcal{J}_{\mathrm{w}}, \mathcal{J}_{\mathrm{b}}$.

Glue the filled Julia sets $\mathcal{K}_{\mathrm{w}}, \mathcal{K}_{\mathrm{b}}$ (of $P_{\mathrm{w}}, P_{\mathrm{b}}$ ) together by identifying $\sigma_{\mathrm{w}}(t) \in \partial \mathcal{K}_{\mathrm{w}}$ with $\sigma_{\mathrm{b}}(-t) \in \partial \mathcal{K}_{\mathrm{b}}$. More precisely, we consider the disjoint union of $\mathcal{K}_{\mathrm{w}}, \mathcal{K}_{\mathrm{b}}$, and let $\mathcal{K}_{\mathrm{w}} \Perp \mathcal{K}_{\mathrm{b}}$ be the quotient obtained from the equivalence relation generated by $\sigma_{\mathrm{w}}(t) \sim \sigma_{\mathrm{b}}(-t)$ for all $t \in \mathbb{R} / \mathbb{Z}$. The minus sign is customary here, though not essential: identifying $\sigma_{\mathrm{w}}(t)$ with $\sigma_{\mathrm{b}}(t)$ amounts to the mating of $P_{\mathrm{w}}$ with $\overline{P_{\mathrm{b}}(\bar{z})}$. The topological mating of $P_{\mathrm{w}}, P_{\mathrm{b}}$ is the map

$$
P_{\mathrm{w}} \Perp P_{\mathrm{b}}: \mathcal{K}_{\mathrm{w}} \Perp \mathcal{K}_{\mathrm{b}} \rightarrow \mathcal{K}_{\mathrm{w}} \Perp \mathcal{K}_{\mathrm{b}},
$$

given by

$$
\left.P_{\mathrm{w}} \Perp P_{\mathrm{b}}\right|_{\mathcal{K}_{i}}=P_{i},
$$

for $i=\mathrm{w}$, b. It follows from (3.3) that it is well defined, namely that $x_{\mathrm{w}} \sim x_{\mathrm{b}} \Rightarrow$ $P_{\mathrm{w}}\left(x_{\mathrm{w}}\right) \sim P_{\mathrm{b}}\left(x_{\mathrm{b}}\right)$ (for all $x_{\mathrm{w}} \in \mathcal{K}_{\mathrm{w}}, x_{\mathrm{b}} \in \mathcal{K}_{\mathrm{b}}$ ).

Definition 3.1. A Thurston map $f: S^{2} \rightarrow S^{2}$ is said to arise as mating if $f$ is topologically conjugate to $P_{\mathrm{w}} \Perp P_{\mathrm{b}}$, the (topological) mating of polynomials $P_{\mathrm{w}}, P_{\mathrm{b}}$.

The Thurston map $g: S^{2} \rightarrow S^{2}$ is equivalent to a mating if $g$ is Thurston equivalent to a Thurston map $f: S^{2} \rightarrow S^{2}$ arising as a mating.

One of the most important result in the theory of matings is the Rees-ShishikuraTan theorem.

Theorem 3.2 ([L.92, Ree92, [Shi00]). Let $P_{\mathrm{w}}=z^{2}+c_{\mathrm{w}}, P_{\mathrm{b}}=z^{2}+c_{\mathrm{b}}$ be two quadratic postcritically finite polynomials, where $c_{\mathrm{w}}, c_{\mathrm{b}}$ are not in conjugate limbs of the Mandelbrot set. Then the topological mating of $P_{\mathrm{w}}, P_{\mathrm{b}}$ exists and is topologically conjugate to a (postcritically finite) rational map (of degree 2).

Recall the a limb of the Mandelbrot set is a component of the complement of the main cardioid.

3.2. Formal mating. We now define the formal mating. Its main purpose is to break up the topological mating into several steps.

We extend $\mathbb{C}$ to the circle at infinity $\left\{\infty \cdot e^{2 \pi i \theta} \mid \theta \in \mathbb{R} / \mathbb{Z}\right\}$, and let $\overline{\mathbb{C}}=$ $\mathbb{C} \cup\left\{\infty \cdot e^{2 \pi i \theta} \mid \theta \in \mathbb{R} / \mathbb{Z}\right\}$. Every monic polynomial $P$ of degree $d$ extends to $\overline{\mathbb{C}}$ by $P\left(\infty \cdot e^{2 \pi i \theta}\right)=\infty \cdot e^{2 \pi i d \theta}$. 
Consider now two monic polynomials $P_{\mathrm{w}}: \overline{\mathbb{C}}_{\mathrm{w}} \rightarrow \overline{\mathbb{C}}_{\mathrm{w}}, P_{\mathrm{b}}: \overline{\mathbb{C}}_{\mathrm{b}} \rightarrow \overline{\mathbb{C}}_{\mathrm{b}}$ of the same degree $d$. Here $\overline{\mathbb{C}}_{\mathrm{w}}=\overline{\mathbb{C}}_{\mathrm{b}}=\overline{\mathbb{C}}$, but we prefer that $P_{\mathrm{w}}, P_{\mathrm{b}}$ are defined on disjoint domains. On the disjoint union $\overline{\mathbb{C}}_{\mathrm{w}} \sqcup \overline{\mathbb{C}}_{\mathrm{b}}$ we define the equivalence relation $\approx$ by identifying $\infty \cdot e^{2 \pi i \theta} \in \overline{\mathbb{C}}_{\mathrm{w}}$ with $\infty \cdot e^{-2 \pi i \theta} \in \overline{\mathbb{C}}_{\mathrm{b}}$. We call the quotient

$$
\overline{\mathbb{C}}_{\mathrm{w}} \uplus \overline{\mathbb{C}}_{\mathrm{b}}:=\overline{\mathbb{C}}_{\mathrm{w}} \sqcup \overline{\mathbb{C}}_{\mathrm{b}} / \approx
$$

the formal mating sphere. Clearly this is a topological sphere. The map

$$
P_{\mathrm{w}} \uplus P_{\mathrm{b}}: \overline{\mathbb{C}}_{\mathrm{w}} \uplus \overline{\mathbb{C}}_{\mathrm{b}} \rightarrow \overline{\mathbb{C}}_{\mathrm{w}} \uplus \overline{\mathbb{C}}_{\mathrm{b}}
$$

given by

$$
P_{\mathrm{w}} \uplus P_{\mathrm{b}}(x)= \begin{cases}P_{\mathrm{w}}(x), & x \in \overline{\mathbb{C}}_{\mathrm{w}} \\ P_{\mathrm{b}}(x), & x \in \overline{\mathbb{C}}_{\mathrm{b}}\end{cases}
$$

is well defined. It is called the formal mating of $P_{\mathrm{w}}, P_{\mathrm{b}}$. The set $\left\{\infty \cdot e^{2 \pi i \theta} \mid \theta \in\right.$ $\mathbb{R} / \mathbb{Z}\} \subset \overline{\mathbb{C}}_{\mathrm{w}} \subset \overline{\mathbb{C}}_{\mathrm{w}} \uplus \overline{\mathbb{C}}_{\mathrm{b}}$ is called the equator of the formal mating sphere.

The ray-equivalence relation is the equivalence relation $\sim$ on the formal mating sphere generated by external rays. Here a closed external ray $R(\theta) \subset \overline{\mathbb{C}}_{\mathrm{w}}$ (where $\theta \in \mathbb{R} / \mathbb{Z}$ ) is one that includes its landing point, as well as its point at infinity, i.e., $\infty \cdot e^{2 \pi i \theta} \in R(\theta)$. Then $\sim$ is the smallest equivalence relation on $\overline{\mathbb{C}}_{\mathrm{w}} \uplus \overline{\mathbb{C}}_{\mathrm{b}}$ such that all points in any closed external ray $R(\theta) \subset \overline{\mathbb{C}}_{\mathrm{w}}$, as well as all points in any closed external ray $R(\theta) \subset \overline{\mathbb{C}}_{\mathrm{b}}$, are equivalent.

It is easy to show that $P_{\mathrm{w}} \uplus P_{\mathrm{b}}$ descends to the quotient $\overline{\mathbb{C}}_{\mathrm{w}} \uplus \overline{\mathbb{C}}_{\mathrm{b}} / \sim$. Moreover this quotient map is topologically conjugate to the topological mating $P_{\mathrm{w}} \Perp P_{\mathrm{b}}$.

We will need the following theorem, originally proved by Rees [Ree92, §1.15]. A more general version was proved by Shishikura [Shi00, Theorem 1.7].

Theorem 3.3. Let $P_{\mathrm{w}}, P_{\mathrm{b}}$ be two hyperbolic, postcritically finite, monic, polynomials, such that the formal mating is Thurston equivalent to a (postcritically finite) rational map $f: \widehat{\mathbb{C}} \rightarrow \widehat{\mathbb{C}}$. Then the topological mating $P_{\mathrm{w}} \Perp P_{\mathrm{b}}$ is topologically conjugate to $f$.

It is well-known that the Julia set of a postcritically finite rational map is locally connected. Furthermore each point in the Julia set of a polynomial is the landing point of only finitely many external rays (this is Douady's lemma, see Shi00, Lemma 4.2]). Assume the quotient $\mathbb{C}_{\mathrm{w}} \uplus \mathbb{C}_{\mathrm{b}} / \sim$, equivalently $\mathcal{K}_{\mathrm{w}} \Perp \mathcal{K}_{\mathrm{b}}$, is a topological sphere. Then each ray equivalence class forms a (actually finite) tree. If this tree does not contain a critical point it is mapped homeomorphically by the formal mating $P_{\mathrm{w}} \uplus P_{\mathrm{b}}$. We have thus the following lemma.

Lemma 3.4. Assume the ray-equivalence relation $\sim$ on the formal mating sphere is given as above. Let $x \in S^{1}$ (i.e., in the equator of the formal mating sphere) and $[x]$ (i.e., the equivalence class of $x$ ) be not a postcritical point of the formal mating $P_{\mathrm{w}} \uplus P_{\mathrm{b}}$. Then the d preimages of $[x]$ by $P_{\mathrm{w}} \uplus P_{\mathrm{b}}$ are given by $[(x+k) / d]$ for $k=0, \ldots, d-1$. The sets $[(x+k) / d]$ are disjoint.

\section{EqUATORS AND HYPERBOLIC RATIONAL MAPS}

Definition 4.1 (equator). Let $f: S^{2} \rightarrow S^{2}$ be a Thurston map, a Jordan curve $\mathcal{E} \subset S^{2} \backslash \operatorname{post}(f)$ is an equator for $f$ if the following three conditions are satisfied.

(1) $\widetilde{\mathcal{E}}:=f^{-1}(\mathcal{E})$ consists of a single component. 
Since $\mathcal{E}$ contains no postcritical point, (1) implies that $\widetilde{\mathcal{E}}$ is a Jordan curve (in $\left.S^{2} \backslash f^{-1}(\operatorname{post}(f)) \subset S^{2} \backslash \operatorname{post}(f)\right)$. Furthermore the degree of the map $f: \widetilde{\mathcal{E}} \rightarrow \mathcal{E}$ is $d=\operatorname{deg} f$.

(2) $\widetilde{\mathcal{E}}$ is isotopic to $\mathcal{E}$ rel. $\operatorname{post}(f)$.

Fix an orientation of $\mathcal{E}$. The curve $\widetilde{\mathcal{E}}$ then inherits an orientation in two distinct ways. First the (covering) map $f: \widetilde{\mathcal{E}} \rightarrow \mathcal{E}$ induces an orientation on $\widetilde{\mathcal{E}}$.

Second, according to (2) let $H_{t}$ be the isotopy rel. post $(f)$ that deforms $\mathcal{E}$ to $\widetilde{\mathcal{E}}$, i.e., $H_{0}=\operatorname{id}_{S^{2}}, H_{1}(\mathcal{E})=\widetilde{\mathcal{E}}$. We choose the orientation on $\widetilde{\mathcal{E}}$ such that (the homeomorphism) $H_{1}: \widetilde{\mathcal{E}} \rightarrow \mathcal{E}$ is orientation-preserving.

(3) $\widetilde{\mathcal{E}}$ is orientation-preserving isotopic to $\mathcal{E}$ rel. post $(f)$.

This means that the two orientations on $\widetilde{\mathcal{E}}$ as given above agree.

If there is an equator $\mathcal{E}$ for the Thurston map $f$, we say that $f$ has an equator. The importance of equators for matings is shown by the following theorem.

Theorem 4.2. A hyperbolic postcritically finite rational map $f$ arises as a mating if and only if $f$ has an equator.

We will need some preparation for the proof of this theorem. There are however many classes of Thurston maps that do not have an equator.

Proposition 4.3. Let $f: S^{2} \rightarrow S^{2}$ be a Thurston map that is not a Thurston polynomial, with \# post $(f) \leq 3$. Then $f$ does not have an equator.

Proof. No Thurston map with \# $\operatorname{post}(f)=0$ or \# post $(f)=1$ exists (see BM11, Remark 5.5]).

If \# post $(f)=2$ then $f$ is Thurston equivalent to $g=z^{m}: \widehat{\mathbb{C}} \rightarrow \widehat{\mathbb{C}}$, where $m \in \mathbb{Z} \backslash\{-1,0,1\}$ (see [BM11, Proposition 6.3]). The case $m \geq 2$ means that $f$ is a Thurston polynomial. In the case $m \leq-2$ there is no equator, since each Jordan curve $\mathcal{C} \subset \widehat{\mathbb{C}} \backslash \operatorname{post}(g)=\mathbb{C} \backslash\{0\}$ which separates the postcritical points $0, \infty$ is isotopic (rel. post $(g)=\{0, \infty\})$ to a small closed loop around 0 . The preimage of such a loop is isotopic rel. $\{0, \infty\}$ to itself, but not orientation-preserving isotopic to itself.

Assume now that post $(f)$ consists of three points, which are denoted for convenience by $0,1, \infty$. Let $\mathcal{E} \subset S^{2} \backslash \operatorname{post}(f)$ be a Jordan curve, which we assume to be not null-homotopic. Then $\mathcal{E}$ is isotopic in $S^{2} \backslash \operatorname{post}(f)$ to a small loop $\mathcal{C}$ around a postcritical point, without loss of generality around $\infty$. Let $\widetilde{\mathcal{C}}:=f^{-1}(\mathcal{C})$. For each $c \in f^{-1}(\infty)$ there is exactly one small loop around $c$ in $\widetilde{\mathcal{C}}$. Thus $\widetilde{\mathcal{C}}$ is a single component if and only if $\infty$ has a single preimage $c$ by $f$. If $c=\infty$ this means that $f$ is a Thurston polynomial. If $c \neq \infty$, this means that $\widetilde{\mathcal{C}}$ is not isotopic rel. $\operatorname{post}(f)$ to $\mathcal{C}$. Thus if $f$ is not a Thurston polynomial it follows that $\mathcal{C}$, hence $\mathcal{E}$, is not an equator of $f$.

Proposition 4.4. Let $f: \widehat{\mathbb{C}} \rightarrow \widehat{\mathbb{C}}$ be a Lattès map. Then $f$ does not have an equator.

Proof. A Lattès map $f$ has three or four postcritical points. In the first case $f$ has no equator by Proposition 4.3 .

In the second case we use the (well-known) explicit description of the map $f$, see [DH93, Proposition 9.3] and [Mil06, Theorem 3.1, Section 4, and Section 5]. 
Namely there is a lattice $\Lambda=\mathbb{Z} \oplus \tau \mathbb{Z}$, where $\tau$ is in the upper half plane. The group $G$ is the subgroup of Aut $(\mathbb{C})$ generated by the translations $z \mapsto z+1, z \mapsto z+\tau$ and the involution $z \mapsto-z$. The quotient map $\wp: \mathbb{C} \rightarrow \mathbb{C} / G$ may be viewed as the Weierstraß $\wp$-function. Finally there is a map $L: \mathbb{C} \rightarrow \mathbb{C}$, given by $L(z)=a z+b$, where $a, b \in \mathbb{C}$. The map $f$ then is topologically conjugate to $L / G: \mathbb{C} / G \rightarrow \mathbb{C} / G$. Here $|a|^{2}=\operatorname{deg} f$. Two cases are possible: either $a \in \mathbb{Z} \backslash\{-1,0,1\}$ or $a \notin \mathbb{R}$ (indeed, there is a more complete description).

Let $\mathcal{E} \subset S^{2} \backslash \operatorname{post}(f)$ be an equator for $f$. Since $f$ is not a Thurston polynomial it follows that $\mathcal{E}$ is not peripheral, i.e., each component of $S^{2} \backslash \mathcal{E}$ contains two postcritical points. The curve $\mathcal{E}$ is isotopic rel. post $(f)$ to a "straight curve" $\mathcal{C}$, i.e., every component of $\widetilde{\mathcal{C}}:=\wp^{-1}(\mathcal{C}) \subset \mathbb{C}$ is a straight line. Note that if two such curves $\mathcal{C}, \mathcal{C}^{\prime}$ lift to lines with distinct slopes, they are not isotopic rel. post $(f)$.

Let $\mathcal{C}^{\prime}:=f^{-1}(\mathcal{C})$. Then the lift by $\wp$ to $\mathbb{C}$ satisfies $\widetilde{\mathcal{C}}^{\prime}:=\wp^{-1}\left(\mathcal{C}^{\prime}\right)=L^{-1}(\widetilde{\mathcal{C}})$.

If $a \in \mathbb{Z} \backslash\{-1,0,1\}$ then $\mathcal{C}$ has $|a|$ preimages by $f$, since $L^{-1}(\wp(\mathcal{C})) / G$ consist of $|a|$ distinct curves. In particular $f^{-1}(\mathcal{C})$, hence $f^{-1}(\mathcal{E})$ does not consist of a single component. Thus $\mathcal{E}$ is not an equator.

If $a \notin \mathbb{R}$ then $\widetilde{\mathcal{C}^{\prime}}=L^{-1}(\widetilde{\mathcal{C}})$ consist of parallel lines which intersect the lines in $\widetilde{\mathcal{C}}$ in the angle $\arg a$. In particular each component of $\widetilde{\mathcal{C}}$ is not isotopic rel. $\operatorname{post}(f)$ to $\mathcal{C}$. Thus $\mathcal{C}$, hence $\mathcal{E}$ is not an equator.

The previous result shows the following. While the existence of an equator is exactly the right condition to check whether a (postcritically finite) hyperbolic rational map arises as a mating (by Theorem 4.2), it is not the right condition in the case when $f$ is not hyperbolic. Namely there are many examples of Lattès maps known which arise as a mating (see [Mil04], Mey11]), yet they do not have an equator by the previous theorem.

Corollary 4.5. A rational map with parabolic orbifold that is not a polynomial does not have an equator.

Proof. A Thurston map $f$ with parabolic orbifold has at most four postcritical points. If $\# \operatorname{post}(f) \leq 3$ the result follows from Proposition 4.3, Every rational map with parabolic orbifold and \# post $(f)=4$ is a Lattès map, where the result follows from Proposition 4.4

Remark 4.6. It is possible to find obstructed Thurston maps with parabolic orbifold that have an equator. Namely the map $z=x+y i \mapsto 2 x+y i$ descends to the quotient by the Weierstraß $\wp$-function. This is an obstructed map with with four postcritical points which has an equator.

The following theorem appeared first in Wittner's thesis [Wit88, Theorem 7.2.1], who credits Thurston. Since Wittner's thesis is not easily available, we provide the proof here.

Theorem 4.7. Let $f: \widehat{\mathbb{C}} \rightarrow \widehat{\mathbb{C}}$ be a postcritically finite rational map, that has an equator $\mathcal{E} \subset S^{2} \backslash \operatorname{post}(f)$. Then $f$ is Thurston equivalent to the formal mating of two polynomials.

Proof. Let $f$ be a postcritically finite rational map, that has an equator. From Corollary 4.5 it follows that $f$ has hyperbolic orbifold, which we assume from now on. 
Let $\mathcal{E}$ be an equator for $f$, and $\widetilde{\mathcal{E}}:=f^{-1}(\mathcal{E})$. Let $\widetilde{U}_{\mathrm{w}}, \widetilde{U}_{\mathrm{b}}$ be the two components of $\widehat{\mathbb{C}} \backslash \widetilde{\mathcal{E}}$. The map $f$ maps $\widetilde{U}_{\mathrm{w}}$, as well as $\widetilde{U}_{\mathrm{b}}$, (properly) to one component of $\widehat{\mathbb{C}} \backslash \mathcal{E}$. Thus we denote the two components $U_{\mathrm{w}}, U_{\mathrm{b}}$ of $\widehat{\mathbb{C}} \backslash \mathcal{E}$ such that $f\left(\widetilde{U}_{\mathrm{w}}\right)=U_{\mathrm{w}}$, $f\left(\widetilde{U}_{\mathrm{b}}\right)=U_{\mathrm{b}}$.

Let $H: \widehat{\mathbb{C}} \times[0,1]$ be an isotopy rel. $\operatorname{post}(f)$ that deforms $\mathcal{E}$ to $\widetilde{\mathcal{E}}$, i.e., $H_{0}=\mathrm{id}_{\widehat{\mathbb{C}}}$ and $H_{1}(\mathcal{E})=\widetilde{\mathcal{E}}$. Then $\widetilde{\mathcal{E}}$ is (forward and backward) invariant for the map $\widetilde{f}: \widehat{\mathbb{C}} \rightarrow \widehat{\mathbb{C}}$ given by $\widetilde{f}=H_{1} \circ f$, i.e., $\widetilde{f}(\widetilde{\mathcal{E}})=\widetilde{\mathcal{E}}=\widetilde{f}^{-1}(\widetilde{\mathcal{E}})$. We can choose the isotopy $H$ such that $\widetilde{f}: \widetilde{\mathcal{E}} \rightarrow \widetilde{\mathcal{E}}$ is topologically conjugate to $z^{d}: S^{1} \rightarrow S^{1}($ where $d=\operatorname{deg} \widetilde{f}=\operatorname{deg} f)$. We will assume this from now on.

Since $\widetilde{\mathcal{E}}$ is orientation-preserving isotopic (rel. post $(f))$ to $\mathcal{E}$, it follows that $H_{1}\left(U_{\mathrm{w}}\right)=\widetilde{U}_{\mathrm{w}}$ and $H_{1}\left(U_{\mathrm{b}}\right)=\widetilde{U}_{\mathrm{b}}$.

On $\widetilde{X}_{\mathrm{w}}:=\widetilde{U}_{\mathrm{w}} \cup \widetilde{\mathcal{E}}$ we consider the equivalence relation which has the following equivalence classes. The set $\widetilde{\mathcal{E}}$ is an equivalence class and every singleton $\{x\} \subset U_{\mathrm{w}}$ is an equivalence class.

Consider the quotient space $S_{\mathrm{w}}^{2}:=\operatorname{clos} \widetilde{U}_{\mathrm{w}} / \widetilde{\mathcal{E}}$. Clearly $S_{\mathrm{w}}^{2}$ is a topological sphere.

The map $H_{1} \circ f$ maps $\widetilde{\mathcal{E}}$ to $\widetilde{\mathcal{E}}$ and each $x \in \widetilde{U}_{\mathrm{w}}$ into $\widetilde{U}_{\mathrm{w}}$. Thus this map descends naturally to the quotient $S_{\mathrm{w}}^{2}$, i.e., to a map $p_{\mathrm{w}}: S_{\mathrm{w}}^{2} \rightarrow S_{\mathrm{w}}^{2}$. Call the point $[\widetilde{\mathcal{E}}] \in S_{\mathrm{w}}^{2}$ for convenience $\infty$. We note that $p_{\mathrm{w}}(\infty)=P_{\mathrm{w}}^{-1}(\infty)=\infty$. Clearly $p_{\mathrm{w}}$ is a postcritically finite branched covering. Thus $p_{\mathrm{w}}$ is a Thurston polynomial.

If $p_{\text {w }}$ would be obstructed it would have a Lévy cycle. Then $f$ would have a Lévy cycle as well. Since we assume that $f$ is a rational map with hyperbolic orbifold this cannot happen. Thus $p_{\mathrm{w}}$ is Thurston equivalent to a polynomial $P_{\mathrm{w}}$, which we assume to be monic and centered. We extend this polynomial to the circle at infinity $S_{\infty}^{1}=\left\{\infty \cdot e^{2 \pi i \theta} \mid \theta \in \mathbb{R} / \mathbb{Z}\right\}$, this extension is still denoted by $P_{\mathrm{w}}: \overline{\mathbb{C}}_{\mathrm{w}} \rightarrow \overline{\mathbb{C}}_{\mathrm{w}}$.

Claim. There are homeomorphisms $h_{0}, h_{1}: \overline{\mathbb{C}}_{\mathrm{w}} \rightarrow \widetilde{X}_{\mathrm{w}}$ with the following properties.

- $h_{0} \circ P_{\mathrm{w}}(z)=\tilde{f} \circ h_{1}(z)$ for all $z \in \overline{\mathbb{C}}_{\mathrm{w}}$, i.e., the following diagram commutes

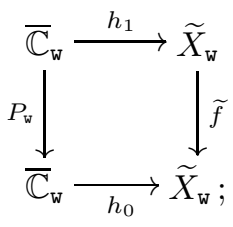

- $h_{0}, h_{1}$ are isotopic rel. $\operatorname{post}\left(P_{\mathrm{w}}\right) \cup S_{\infty}^{1}$, in particular $h_{0}=h_{1}$ on $S_{\infty}^{1}$.

Proof of Claim. We will deform $P_{\mathrm{w}}: \overline{\mathbb{C}}_{\mathrm{w}} \rightarrow \overline{\mathbb{C}}_{\mathrm{w}}$ by an isotopy rel. $\operatorname{post}\left(P_{\mathrm{w}}\right) \cup S_{\infty}^{1}$ and $\widetilde{f}: \widetilde{X}_{\mathrm{w}} \rightarrow \widetilde{X}_{\mathrm{w}}$ by an isotopy rel. $\operatorname{post}(\widetilde{f}) \cup \widetilde{\mathcal{E}}$. It is enough to prove the statement with these deformed maps.

More precisely, we can postcompose $P_{\mathrm{w}}$ with an isotopy rel. $\operatorname{post}\left(P_{\mathrm{w}}\right) \cup S_{\infty}^{1}$ such that the resulting map is topologically conjugate to $\varphi: S^{1} \times[0,1] \rightarrow S^{1} \times[0,1]$, $\varphi(\theta, t)=(d \theta, t)$ in an annulus $A$ containing $S_{\infty}^{1} \subset \overline{\mathbb{C}}_{\mathrm{w}}$. We call this deformed map $\widetilde{P}_{\mathrm{w}}$. The boundary component of $A$ distinct from $S_{\infty}^{1}$ is called $\mathcal{C}$. Note that $\widetilde{P}_{\mathrm{w}}(\mathcal{C})=\mathcal{C}$.

Since $P_{\mathrm{w}}$, hence $\widetilde{P}_{\mathrm{w}}$, is Thurston equivalent to $p_{\mathrm{w}}$, it follows that there are homeomorphisms $k_{0}, k_{1}: \mathbb{C}_{\mathrm{w}} \rightarrow \widetilde{U}_{\mathrm{w}}$ such that $k_{0} \circ \widetilde{P}_{\mathrm{w}}=\widetilde{f} \circ k_{1}$ on $\mathbb{C}_{\mathrm{w}}$, that are isotopic rel. $\operatorname{post}\left(P_{\mathrm{w}}\right)$. 
Let $\widetilde{\mathcal{C}}:=k_{0}(\mathcal{C}) \subset \widetilde{U}_{\mathrm{w}}$ and $\widetilde{\mathcal{C}}^{1}:=\widetilde{f}^{-1}(\mathcal{C})=k_{1}(\mathcal{C})$. We can precompose $\widetilde{f}$ with a pseudo-isotopy $I$ rel. post $(\widetilde{f}) \cup \widetilde{\mathcal{E}}$, so that the resulting map $\widetilde{f} \circ I_{1}$ leaves $\widetilde{\mathcal{C}}$ invariant, and $\widetilde{f} \circ I_{1}$ is topologically conjugate to $\varphi$ as above. Let $\widetilde{A}$ be the annulus between $\mathcal{E}$ and $\widetilde{\mathcal{C}}$.

We define $h_{0}=k_{1}$ on $\mathbb{C}_{\mathrm{w}} \backslash A$ and $h_{1}=I_{1} \circ k_{1}$ on $\mathbb{C}_{\mathrm{w}} \backslash A$. Since both $\widetilde{P}_{\mathrm{w}}$ and $\widetilde{f} \circ I_{1}$ are topologically conjugate to $\varphi$ on $A$ respectively $\widetilde{A}$, we can extend $h_{0}, h_{1}$ to $A$ (mapping to $\widetilde{A}$ ) such that the claim holds.

In the same fashion we define $S_{\mathrm{b}}^{2}:=\operatorname{clos} \widetilde{U}_{\mathrm{b}} / \widetilde{\mathcal{E}}$ and the map $p_{\mathrm{b}}: S_{\mathrm{b}}^{2} \rightarrow S_{\mathrm{b}}^{2}$. Again this is a Thurston polynomial, which is Thurston equivalent to a (monic, centered) polynomial $P_{\mathrm{b}}$ (i.e., is not obstructed). The analog statement to the claim above holds for $P_{\mathrm{b}}$.

It is not necessarily true that $\tilde{f}$ (hence $f$ ) is Thurston equivalent to the formal mating of $P_{\mathrm{w}}, P_{\mathrm{b}}$. However the maps $h_{0}, h_{1}$ in the above claim have to map one of the $d-1$ fixed points of $P_{\mathrm{w}}$ on $S_{\infty}^{1} \subset \overline{\mathbb{C}}_{\mathrm{w}}$ to a fixed point of $\tilde{f}$ on $\mathcal{E}$. Similarly for $P_{\mathrm{b}}$. Thus $\widetilde{f}$, hence $f$, is Thurston equivalent to the formal mating of $P_{\mathrm{w}}$ and $c^{-1} P_{\mathrm{b}}(c z)$, where $c=e^{2 \pi i j /(d-1)}$, for some $j=0, \ldots d-2$.

Proof of Theorem 4.2. Let $f: \widehat{\mathbb{C}} \rightarrow \widehat{\mathbb{C}}$ be a hyperbolic postcritically finite rational map.

Assume $f$ arises as a mating. Let $S^{2}=\overline{\mathbb{C}}_{\mathrm{w}} \uplus \overline{\mathbb{C}}_{\mathrm{b}}$ be the formal mating sphere with equator $S^{1}:=\left\{\infty \cdot e^{2 \pi i \theta} \mid \theta \in[0,2 \pi]\right\} \subset \overline{\mathbb{C}}_{\mathrm{w}} \subset S^{2}$ and $\sim$ be the ray-equivalence on $S^{2}$. Let $H: S^{2} \times I \rightarrow S^{2}$ be the pseudo-isotopy realizing $\sim$ according to Moore's Theorem.

We fix an appropriate $\epsilon>0$ such that for $t \in[1-\epsilon, 1]$ the curve $H\left(S^{1}, t\right)$ does not meet post $(f)$, i.e., $H\left(S^{1} \times[1-\epsilon, 1]\right) \subset S^{2} \backslash \operatorname{post}(f)$. Consider the two parametrized curves $\gamma_{1}, \gamma_{1-\epsilon}: S^{1} \rightarrow S_{f}^{2}$ given by $\gamma_{1}\left(\infty \cdot e^{2 \pi i \theta}\right)=H_{1}\left(\infty \cdot e^{2 \pi i d \theta}\right)$, and more generally $\gamma_{t}\left(\infty \cdot e^{2 \pi i \theta}\right)=H_{t}\left(\infty \cdot e^{2 \pi i d \theta}\right)$ for each $t \in[1-\epsilon, 1]$ where $d=\operatorname{deg} f$. Note that they cover the image (at least) $d$-fold. Clearly $H: S^{1} \times[1-\epsilon, 1] \rightarrow S^{2}$ is a homotopy deforming $\gamma_{1-\epsilon}$ to $\gamma_{1}$.

Consider now $\widetilde{\gamma}_{1}: S^{1} \rightarrow S^{2}$ given by $\widetilde{\gamma}_{1}\left(\infty \cdot e^{2 \pi i \theta}\right):=H_{1}\left(e^{2 \pi i \theta}\right)$. By (3.3) $\widetilde{\gamma}_{1}$ is a lift of $\gamma_{1}$ by $f$, i.e., $f \circ \widetilde{\gamma}\left(\infty \cdot e^{2 \pi i \theta}\right)=\gamma\left(\infty \cdot e^{2 \pi i \theta}\right)$. By the standard lifting theorem of homotopies by covering maps it follows that the homotopy $H: S^{1} \times[1-\epsilon, 1] \rightarrow S^{2}$ can be lifted by $f$ to a homotopy $\widetilde{H}: S^{1} \times[1-\epsilon, 1] \rightarrow S^{2}$ with $\widetilde{H}_{1}=\widetilde{\gamma}_{1}$, i.e., $f \circ \widetilde{H}=H$. Let $\widetilde{\gamma}_{t}: S^{1} \rightarrow S^{2}$ be given by $\widetilde{\gamma}_{t}\left(\infty \cdot e^{2 \pi i \theta}\right):=\widetilde{H}_{t}\left(\infty \cdot e^{2 \pi i \theta}\right)$. Thus $f \circ \widetilde{\gamma}_{t}\left(\infty \cdot e^{2 \pi i \theta}\right)=\gamma_{t}\left(\infty \cdot e^{2 \pi i \theta}\right)$. Thus $\widetilde{\gamma}_{t}$ is a component of $f^{-1}\left(\gamma_{t}\right)$.

It remains to show that there is no other component, i.e., that $\widetilde{\gamma}_{t}$ is the whole preimage of $\gamma_{t}$ by $f$. This is seen using Lemma 3.4. Indeed for any $\gamma_{1}\left(\infty \cdot e^{2 \pi i \theta}\right)=$ $H_{1}\left(\infty \cdot e^{2 \pi i d \theta}\right)$ the points $H_{1}\left(\infty \cdot e^{2 \pi i \theta+\frac{k}{d}}\right), k=0, \ldots, d-1$ are the $d$ distinct preimages by $f$. By continuity $\widetilde{\gamma}_{t}\left(\infty \cdot e^{2 \pi i \theta+\frac{k}{d}}\right), k=0, \ldots, d-1$ are distinct for $t$ sufficiently close to 1 . Thus $\widetilde{\gamma}_{t}$ is the whole preimage of $\gamma_{t}$ by $f$.

Since $\mathcal{C}_{t}:=\gamma_{t}\left(S^{1}\right)$ contains no postcritical point $\widetilde{\mathcal{C}}_{t}:=\widetilde{\gamma}_{t}\left(S^{1}\right)$ is a simple curve. Finally the concatenation of $H$ and $\widetilde{H}$ deforms $\mathcal{C}_{t}$ to $\widetilde{\mathcal{C}_{t}}$, thus they are homotopic rel. post $(f)$. Clearly each component of $S^{2} \backslash \mathcal{C}_{t}$ contains at least a postcritical point. Thus $\mathcal{C}_{t}, \widetilde{\mathcal{C}}_{t}$ are orientation-preserving isotopic rel. post $(f)$ by Theorem 2.6. 
Assume now that $f$ has an equator. Then $f$ is Thurston equivalent to the formal mating of two polynomials $P_{\mathrm{w}}, P_{\mathrm{b}}$ by Theorem 4.7. From the Rees-Shishikura theorem, i.e., Theorem [3.3, it follows that $f$ is topologically conjugate to (the topological mating) $P_{\mathrm{w}} \Perp P_{\mathrm{b}}$.

Theorem 4.2 together with Proposition 4.3 immediately yields the following.

Theorem 4.8. Let $f: \widehat{\mathbb{C}} \rightarrow \widehat{\mathbb{C}}$ be a hyperbolic, postcritically finite, rational map, with \# post $(f)=3$, that is not a polynomial. Then $f$ does not arise as a mating.

In the case when $f$ is a polynomial it arises trivially as the mating of itself with $z^{d}$ (where $d=\operatorname{deg} f$ ). Note that each iterate $f^{n}$ has the same postcritical set. Thus in the case of the previous theorem no iterate $f^{n}$ arises as mating. This of course is in contrast to the results from Mey11 and [Mey].

\section{An Example}

We provide an example of a rational map to be able to illustrate the following. We give several descriptions (naturally equivalent) of the same map.

Consider two equilateral triangles. We glue them together along their boundary. This yields a topological sphere denoted by $\Delta$. We color one side (i.e., one of the equilateral triangles) white and call it $T_{\mathrm{w}}$, the other one is colored black and called $T_{\mathrm{b}}$. We call $T_{\mathrm{w}}, T_{\mathrm{b}}$ the 0 -triangles.

Glue again two equilateral triangles (of the same size as before) together to form a topological sphere $\Delta^{1}$ as before. Each face is now divided into four equilateral triangles of half the side-length. We color these small triangles in a checkerboard pattern black and white. This means that two small triangles which share an edge have different color. These small triangles are called 1-triangles.

Consider a small white triangle $T_{1} \subset \Delta^{1}$. The map $f$ is given on $T_{1}$ as follows. Scale $T_{1}$ by the factor 2 and map it to the big white triangle in $\Delta$. Consider now a small black triangle $T_{2} \subset \Delta^{1}$ that intersects $T_{1}$ in an edge. The map $f$ can be extended continuously to $T_{2}$ by scaling by the factor 2 and mapping it to the black triangle in $\Delta$.

Continuing in this fashion we obtain a map $\Delta^{1} \rightarrow \Delta$. Identifying $\Delta^{1}$ with $\Delta$ we obtain a branched covering map from a topological sphere to itself. Note that the critical points (i.e., the points where $f$ is not locally injective) are the vertices of the small triangles. These are mapped to the vertices of $\Delta$, each such vertex is mapped to (possibly) another vertex of $\Delta$. Thus the map is postcritically finite.

The map may not look as a rational map to many readers. To the reader familiar with Thurston's classification of rational maps among Thurston maps (see [DH93]) we remark that $f$ has only 3 postcritical points, thus it has no Thurston obstruction (the orbifold of $f$ though is parabolic).

There is however a standard way to view every polyhedral surface as a Riemann surface (see Bea84]). We outline the construction in the case at hand.

Consider first a point $p \in \Delta$ that lies in the interior of either one of the two triangles from which $\Delta$ is built, say $p \in \operatorname{int} T_{\mathrm{w}}$. Then (any) orientation-preserving, isometric map from $\operatorname{int} T_{\mathrm{w}}$ to (the interior of) an equilateral triangle in the plane is a chart.

Assume now that $p$ lies on a common edge $E$ of $T_{\mathrm{w}}$ and $T_{\mathrm{b}}$, but is not a vertex of $\Delta$. Then the map to (the interior of) the union of two equilateral triangles in the 


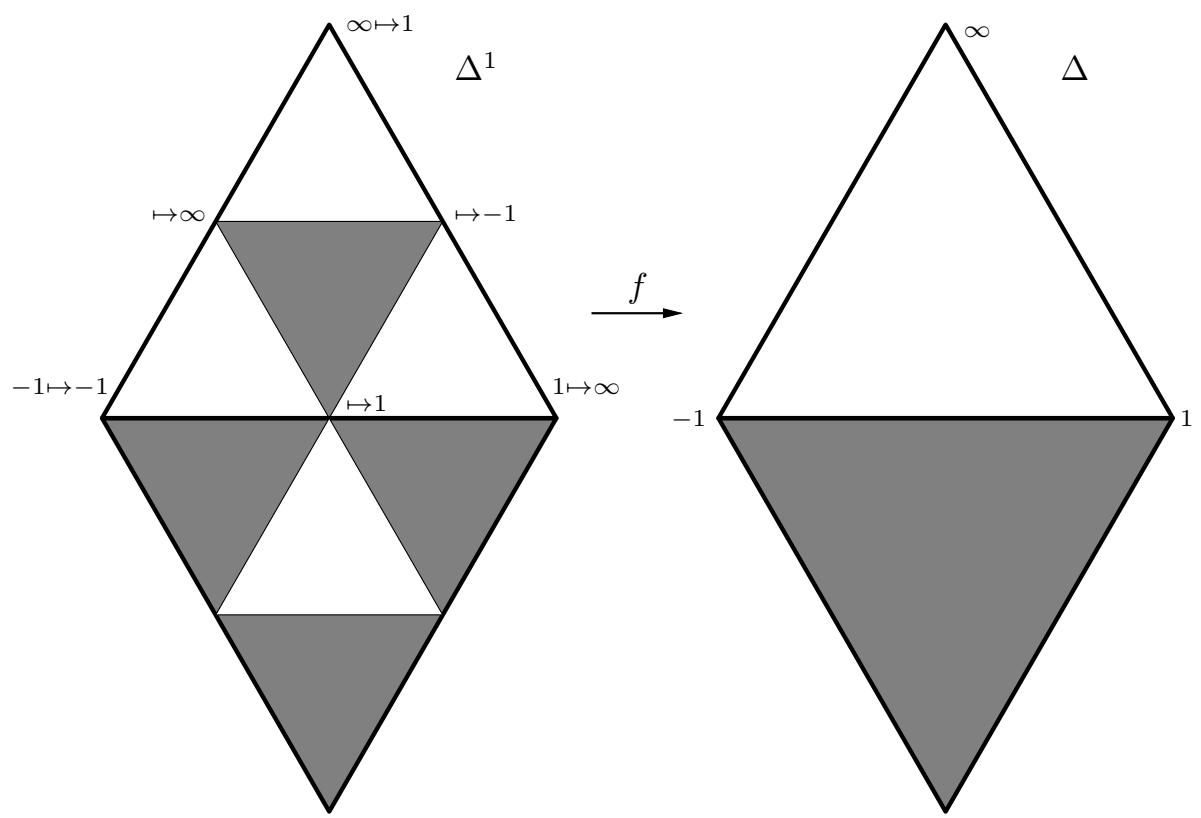

Figure 1. A Lattès map with signature $(3,3,3)$.

plane is a chart. Here of course we demand that this chart is orientation preserving, and the interiors of $T_{\mathrm{w}}, T_{\mathrm{b}}, E$ are mapped isometrically.

Finally if $p$ is a vertex of $\Delta$ we note that the (Euclidean) total angle around $p$ is $2 \pi / 3$. Thus we can map a small open neighborhood of $p$ in $\Delta$ essentially by the map $z \mapsto z^{3}$ to a planar domain.

Changes of coordinates are conformal, thus we have defined a Riemann surface. Clearly this is compact and simply connected, thus conformally equivalent to the Riemann sphere $\widehat{\mathbb{C}}$. Note that the map $f: \Delta \rightarrow \Delta$ is holomorphic with respect to these charts. Thus changing the coordinate system yields a holomorphic, hence a rational map $f: \widehat{\mathbb{C}} \rightarrow \widehat{\mathbb{C}}$.

A slightly different way to construct the map may be thought of as constructing the uniformizing map above explicitly. Namely map the equilateral triangle $T_{\mathrm{w}}$ to the upper half-plane conformally, i.e., by a Riemann map $\varphi$ normalized such that the three vertices are mapped to $-1,1, \infty$. Recall that $T_{\mathrm{w}}$ is subdivided into four triangles of half the side-length. Consider one (of the three) such triangles which is colored white $T_{1}$. We consider the image in the upper half-plane, i.e., $T_{1}^{\prime}:=\varphi\left(T_{1}\right) \subset \operatorname{clos} \mathbb{H}^{+}$.

We map $T_{1}^{\prime}$ conformally to the upper half-plane, such that the images of the vertices by $\varphi$ are mapped to $-1,1, \infty$. Figure 1 indicates which vertices are mapped to which of the points $-1,1, \infty$.

Consider now the black 1-triangle $T_{2} \subset T_{\text {w }}$. It shares an edge $E$ with $T_{1}$. Its image is $T_{2}^{\prime}:=\varphi\left(T_{2}\right)$.

Note that $T_{2}$ is the reflection of $T_{1}$ along $E$. Thus $T_{2}^{\prime}$ is the conformal reflection of $T_{1}^{\prime}$ along $E^{\prime}:=\varphi(E)$. By the Schwarz reflection principle it follows that the map $f: T_{1}^{\prime} \rightarrow \operatorname{clos} \mathbb{H}^{+}$extends conformally to $T_{2}^{\prime}$ and maps this set to the lower half-plane (vertices are mapped to $-1,1, \infty$ ). 
Continuing in this fashion we construct a map $f: \operatorname{clos} \mathbb{H}^{+}=\varphi\left(T_{\mathrm{w}}\right) \rightarrow \widehat{\mathbb{C}}$. There are now two ways to proceed. Either we map $T_{\mathrm{b}}$ conformally to the lower half-plane and proceed as before. Alternatively we note that the map $f: \operatorname{clos} \mathbb{H}^{+}=\varphi\left(T_{\mathrm{w}}\right) \rightarrow \widehat{\mathbb{C}}$ maps the extended real line $\widehat{\mathbb{R}}=\mathbb{R} \cup\{\infty\}$ to itself. Thus we can use the Schwarz reflection principle again to extend $f$ to the lower half-plane via $f(z):=\overline{f(\bar{z})}$.

A third description to construct the map $f$ is as follows. Consider the triangular lattice $\Lambda:=\mathbb{Z} \oplus \omega \mathbb{Z}$, where $\omega=\exp (\pi i / 3)$. Map the equilateral triangle with vertices $0,1, \omega$ by a Riemann map $\wp$ to the upper half-plane, such that $\wp(0)=$ $-1, \wp(1)=1, \wp(\omega)=\infty$. This map extends by reflection to a holomorphic map $\wp: \mathbb{C} \rightarrow \widehat{\mathbb{C}}$. It is the Weierstraß $\wp$-function to the lattice $\Lambda$ (slightly differently normalized than usual). Consider now the map $z \mapsto 2 z$ on $\mathbb{C}$. It is straightforward to check that if $z, w \in \mathbb{C}$ are mapped by $\wp$ to the same point, then the same is true for $2 z, 2 w$. Thus there is a well defined map $f: \widehat{\mathbb{C}} \rightarrow \widehat{\mathbb{C}}$ (which is the same map as before) such that the diagram

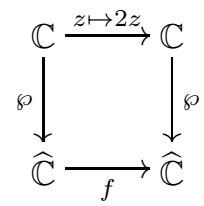

commutes. Finally we note that the map $f$ is given by

$$
f=\frac{2(z+1)(z-3)^{3}}{(z-1)(z+3)^{3}}-1 .
$$

\section{A SUFFICIENT CRITERION FOR MATING}

In Section 4 we saw a necessary and sufficient criterion for a hyperbolic postcritically finite rational map to arise as a mating.

Here we present another sufficient criterion for a map to arise as a mating from Mey11 and Mey.

Recall that for an equator we required the existence of a Jordan curve in $\widehat{\mathbb{C}} \backslash$ $\operatorname{post}(f)$ that can be deformed by a isotopy rel. post $(f)$ orientation-preserving to its preimage.

The existence of an equator is the right condition to check whether $f$ arises as a mating for hyperbolic maps. It is not the right condition however, for maps that are not hyperbolic. Here we concentrate on the case that the map $f$ is as far away as possible from being hyperbolic, i.e., on the case where all critical points, hence all postcritical points are in the Julia set.

Whereas in the hyperbolic case, we considered Jordan curves avoiding the postcritical set, we consider here Jordan curves containing the postcritical set. So let $\mathcal{C} \subset \widehat{\mathbb{C}}$ be a Jordan curve with post $(f) \subset \mathcal{C}$. The postcritical points divide $\mathcal{C}$ into closed Jordan arcs, which are called 0-edges.

We consider the preimage

$$
\mathcal{C}^{1}:=f^{-1}(\mathcal{C}) .
$$

This set can be naturally viewed as a graph embedded in the sphere. Namely the set $\mathbf{V}^{1}:=f^{-1}(\operatorname{post}(f))$ is the set of vertices of this graph, the closure of one component of $f^{-1}(\mathcal{C}) \backslash \mathbf{V}^{1}$ is an edge (called a 1-edge) of this graph. All edges arise 
in this form. We call each point $p \in \mathbf{V}^{1}$ a 1-vertex. Note that every postcritical point, as well as every critical point, is a 1-vertex.

There may be multiple edges connecting two vertices, but there can be no loops. For each 1-edge $E^{1} \subset f^{-1}(\mathcal{C})$ there is a 0-edge $E^{0} \subset \mathcal{C}$ such that the map $f: E^{1} \rightarrow$ $E^{0}$ is a homeomorphism.

Note that each critical point $c$ is incident to $2 \operatorname{deg}_{f}(c) 1$-edges. In particular $\mathcal{C}^{1}$ is not a Jordan curve, thus cannot be isotopic to $\mathcal{C}$.

Roughly speaking we demand that there is a pseudo-isotopy rel. post $(f)$ that deforms $\mathcal{C}$ to $f^{-1}(\mathcal{C})$.

To complete the picture, we let $X_{\mathrm{w}}^{0}, X_{\mathrm{b}}^{0}$ be the closures of the two components of $\widehat{\mathbb{C}} \backslash \mathcal{C}$. Then $X_{\mathrm{w}}^{0}$ will be colored white, and $X_{\mathrm{b}}^{0}$ is colored black. They are called the two 0 -tiles. We orient $\mathcal{C}$, so that it is positively oriented as boundary of the white 0 -tile $X_{\mathrm{w}}^{0}$.

The closure of each component of $\widehat{\mathbb{C}} \backslash \mathcal{C}^{1}$ is called a 1-tile. It is not very hard to show that each 1-tile $X$ is mapped by $f$ homeomorphically to either $X_{\mathrm{v}}^{0}$ or $X_{\mathrm{b}}^{0}$, see [BM11, Chapter 4.3]. In the first case we color $X$ white, in the second black.

Recall the definition of a pseudo-isotopy from Definition 2.3. There are many "bad" ways in which $\mathcal{C}$ be deformed by a pseudo-isotopy to $f^{-1}(\mathcal{C})$. For example a whole interval or a Cantor set may be deformed to a postcritical point. Also some arc $A \subset \mathcal{C}$ may be deformed to some edge in $f^{-1}(\mathcal{C})$ in a highly non-trivial fashion.

Definition 6.1. An elementary, orientation-preserving, pseudo-isotopic deformation of $\mathcal{C}$ to $\mathcal{C}^{1}=f^{-1}(\mathcal{C})$ rel. $\operatorname{post}(f)$ is a pseudo-isotopy $H: S^{2} \times[0,1]$ rel. $\operatorname{post}(f)$ (with $H_{0}=\mathrm{id}_{S^{2}}$ ) such that

(1) $H_{1}(\mathcal{C})=\mathcal{C}^{1}$

(2) the set of points $w \in \mathcal{C}$ such that $H_{1}(w)$ is a 1-vertex is finite. The set of all such points is denoted by $\mathbf{W}:=\left(H_{1}\right)^{-1}\left(\mathbf{V}^{1}\right) \cap \mathcal{C}$. Note that post $(f) \subset \mathbf{W}$;

(3) restricted to $\mathcal{C} \backslash \mathbf{W}$ the homotopy $H$ is an isotopy, i.e.,

$$
H_{1}: \mathcal{C} \backslash \mathbf{W} \rightarrow \mathcal{C}^{1} \backslash \mathbf{V}^{1} \text { is a homeomorphism. }
$$

This means there is a bijection between 1-edges and closures of components of $\mathcal{C} \backslash \mathbf{W}$. Furthermore if $E^{1} \subset \mathcal{C}^{1}$ is a 1-edge and $A \subset \mathcal{C}$ is the corresponding arc (i.e., closure of a component of $\mathcal{C} \backslash \mathbf{W}$ ), then $H_{1}: A \rightarrow E^{1}$ is a homeomorphism.

(4) we assign an orientation to $\mathcal{C}$. For each 1-edge $E^{1} \subset \mathcal{C}^{1}$ there are two ways to map it homeomorphically to (a part of) $\mathcal{C}$. Namely by

$$
H_{1}: A \rightarrow E^{1} \text { and by } f: E^{1} \rightarrow E^{0} .
$$

Here $A \subset \mathcal{C}$ is a (closed) arc corresponding to $E^{1}$ as above, $E^{0} \subset \mathcal{C}$ is a 0 edge. We demand that $H$ deforms $\mathcal{C}$ orientation-preserving to $\mathcal{C}^{1}$, meaning that the orientations on $E^{1}$ induced by the two homeomorphisms above agree for each 1-edge.

If there is a pseudo-isotopy $H$ for $\mathcal{C}$ as above, we say that $f$ has a pseudo-equator.

Remark 6.2. Let the pseudo-isotopy $H: S^{2} \times[0,1] \rightarrow S^{2}$ be as above. Then for any point $p \in \mathcal{C}^{1} \backslash \mathbf{V}^{1}$ there is exactly one point $q \in \mathcal{C}$ that is mapped by $H_{1}$ to $p$. For a point $c \in \mathbf{V}^{1}$ there are exactly $\operatorname{deg}_{f}(c)$ points in $\mathcal{C}$ that are mapped by $H_{1}$ to $c$. In this sense the curve $\mathcal{C}$ is deformed by $H_{1}$ to $\mathcal{C}^{1}$ as simply as possible.

The following is proved in Mey11 and Mey. 


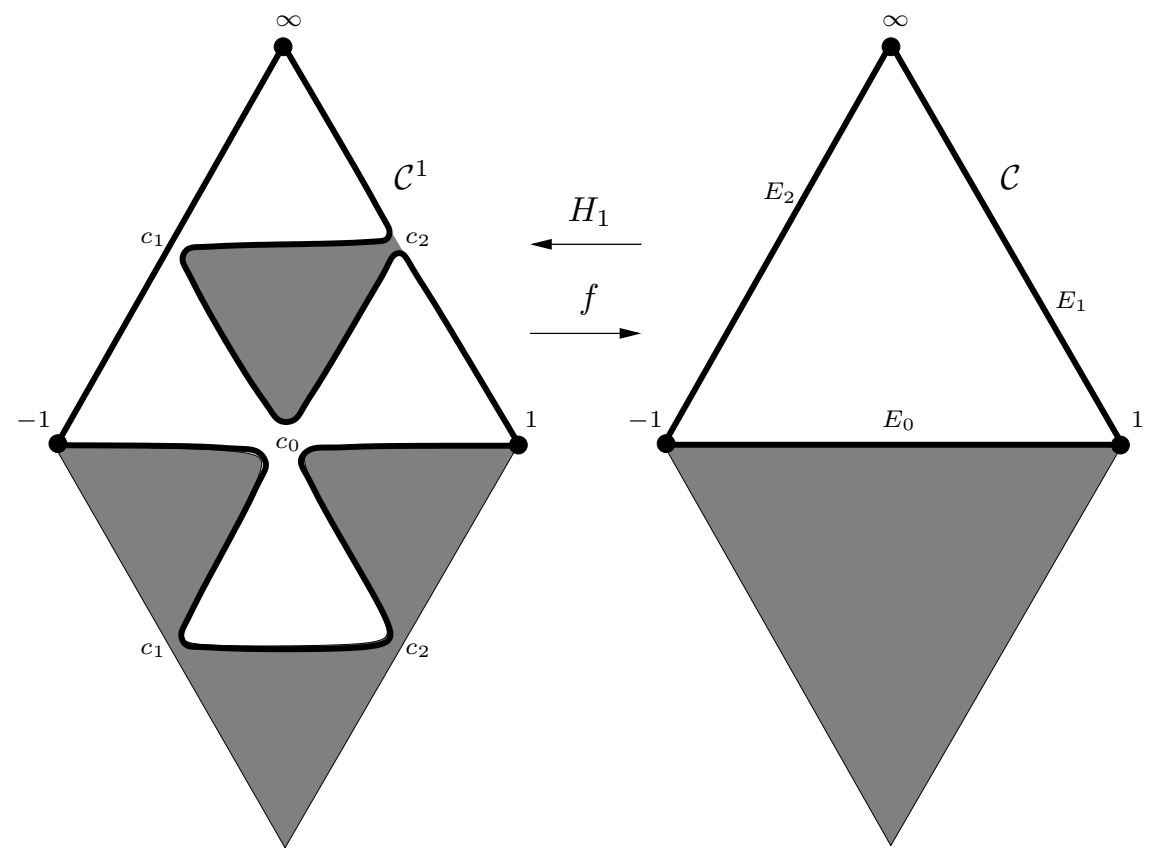

Figure 2. Deforming $\mathcal{C}$ to $\mathcal{C}^{1}$.

Theorem 6.3. Let $f: \widehat{\mathbb{C}} \rightarrow \widehat{\mathbb{C}}$ be a postcritically finite rational map with Julia set $\mathcal{J}(f)=\widehat{\mathbb{C}}$. Assume $f$ has a pseudo-equator as in Definition 6.1. Then $f$ arises as a (topological) mating of two (postcritically finite, monic) polynomials $P_{\mathrm{w}}, P_{\mathrm{b}}$.

Remark 6.4. The theorem above remains true in the case when the map $f$ is not a rational map. Namely it holds for expanding Thurston maps. The statement has to be slightly modified in the presence of periodic critical points.

Example 6.5. We consider the example from Section [5. Recall that $\operatorname{post}(f)=$ $\{-1,1, \infty\}$. These points are the vertices of the pillow in Figure 1. We choose $\mathcal{C}=\widehat{\mathbb{R}}$. In the model of the map indicated in Figure 1, $\mathcal{C}$ is the common boundary of the two triangles which forms the pillow. The preimage $\mathcal{C}^{1}:=f^{1}(\mathcal{C})$ is the union of all the edges of the small triangles to the left in Figure 1 .

The pseudo-isotopy $H$ that deforms $\mathcal{C}$ elementary, orientation-preserving, to $\mathcal{C}^{1}=$ $f^{-1}(\mathcal{C})$ is indicated (to the left) in Figure 2,

Example 6.6. We consider again the same example from Section 5 Again $\mathcal{C}=\widehat{\mathbb{R}}$. We have chosen to draw the curve $\mathcal{C}$ on the right of Figure 3 as the boundary of the black 0-tile, however. In Figure 3 we show a pseudo-isotopy that deforms $\mathcal{C}$ to $\mathcal{C}^{1}$ in an orientation-reversing way. By this we mean that the shown pseudo-isotopy $H$ satisfies all properties from Definition 6.1 except (44). This is seen as follows. If we traverse $\mathcal{C}$ positively as boundary of the white 0 -tile, we go through the postcritical points in the cyclic order $-1 \rightarrow 1 \rightarrow \infty \rightarrow-1$. However if we traverse $\mathcal{C}^{1}:=f^{-1}(\mathcal{C})$ in the orientation given by $f$ (i.e., each 1-edge is traversed positively as boundary of the white 1-tile in which it is contained), then the postcritical points are traversed in the order $-1, \infty, 1$. More precisely, we see that the orientations on each 1-edge $E^{1}$ induced by $f$ and by $H_{1}$ are opposite. 


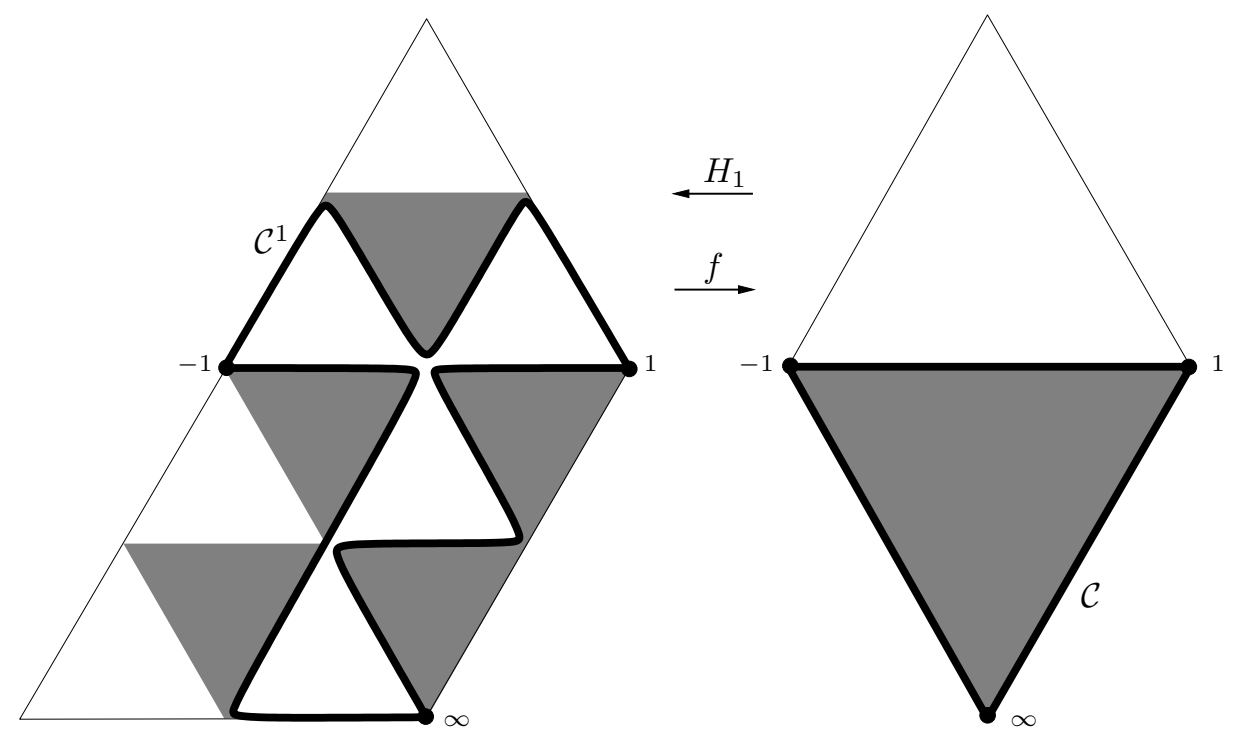

FiguRE 3. Orientation reversing pseudo-isotopy.

If we compare Figure 2 with Figure 3 we see one difference. Namely in the pseudo-isotopy indicated in Figure 2 the interior of $\mathcal{C}$, more precisely the interior of the white 0 -tile $X_{\mathrm{w}}^{0}$, is deformed to all white 1-tiles. On the other hand the pseudo-isotopy indicated in Figure 3 deforms the interior of the black 0-tile to the white 1-tiles. This is a general phenomenon.

Lemma 6.7. Let $H$ be an elementary, pseudo-isotopic deformation of $\mathcal{C}$ to $\mathcal{C}^{1}$, i.e., a pseudo-isotopy rel. post $(f)$ that satisfies (1), (2), and (3) (but not necessarily (4)) of Definition 6.1. Then

(1) Either $H$ deforms $\mathcal{C}$ to $\mathcal{C}^{1}$ orientation-preserving (i.e., satisfies (4) of Definition [6.1), or $H$ deforms $\mathcal{C}$ to $\mathcal{C}^{1}$ orientation-reserving. The latter means that for any 1-edge the orientations induced by the homeomorphisms

$$
f: E^{1} \rightarrow E^{0} \text { and } H_{1}: A \rightarrow E^{1}
$$

disagree. Here $E^{0} \subset \mathcal{C}$ is a 0 -edge and $A \subset \mathcal{C}$ is an arc as in Definition 6.1 (3).

Let $x, y \in\left(H_{1}\right)^{-1}\left(\mathcal{C}^{1}\right)$, i.e., two points that are mapped by $H_{1}$ to the interior of (possibly distinct) 1-tiles. Let $X^{0} \ni x, Y^{0} \ni y$ be the 0-tiles containing $x, y$, and $X^{1} \ni H_{1}(x), Y^{1} \ni H_{1}(y)$ be the 1 -tiles containing $H_{1}(x), H_{1}(y)$. Then

(2)

$$
\begin{aligned}
& X^{0}, Y^{0} \text { have the same color } \\
\Longleftrightarrow & X^{1}, Y^{1} \text { have the same color. }
\end{aligned}
$$

$$
X^{0} \text { and } X^{1} \text { have the same color }
$$$$
\Longleftrightarrow H \text { is orientation-preserving, }
$$

i.e., $H$ satisfies (4) of Definition 6.1. 
Proof. (11) Consider a critical point, i.e., a 1-vertex $c$. This point is contained in the 1-edges $E_{0}, \ldots, E_{n-1}$ (where $n=2 \operatorname{deg}_{f}(c)$ ), which are labeled mathematically positively around $c$.

We first note that the orientation on these 1-edges induced by $f$ alternates. This is seen as follows. There are $n$ black as well as $n$ white 1-tiles around $c$, the colors of the 1-tiles around $c$ alternate. The endpoint of the edge $E_{j}$ is $c$ (via the orientation given by $f$ ) if and only if the sector between $E_{j-1}, E_{j}$ contains a white 1-tile (and the sector between $E_{j}, E_{j+1}$ contains a black 1-tile). Conversely the initial point of $E_{j}$ is $c$ if and only if the sector between $E_{j-1}, E_{j}$ contains a black 1-tile (and the sector between $E_{j}, E_{j+1}$ contains a white 1-tile).

We now consider the orientation on 1-edges induced by the pseudo-isotopy $H$.

Let $A, A^{\prime} \subset \mathcal{C}$ be two adjacent arcs that are deformed to 1-edges $E, E^{\prime}$ as in Definition 6.1 (3), where $A \cap A^{\prime}$ is deformed to $c$ by $H$. We assume that $A^{\prime}$ succeeds $A$ with respect to the orientation of $\mathcal{C}$. Then (with respect to the orientation induced by $H$ ) the endpoint of $E$ is $c$, while $c$ is the initial point of $E^{\prime}$.

Since $H$ is a pseudo-isotopy $H_{1-\epsilon}$ is a homeomorphism for all $0<\epsilon<1$. It follows that in the sector between $E, E^{\prime}$ there is an even number of 1-edges.

Thus from the first claim it follows that the orientation on $E$ induced by $H$ agrees with the orientation on $E$ induced by $f$ if and only if the orientations on $E^{\prime}$ induced by $H$ and $f$ agree.

Consider the $\operatorname{arc} A^{\prime \prime}$ on $\mathcal{C}$ that succeed $A^{\prime}$. This is deformed by $H$ to a 1-edge $E^{\prime \prime}$. The orientations induced on $E^{\prime \prime}$ by $f$ and $H$ agree if and only if the respective orientations on $E^{\prime}$ agree. Continuing in this fashion we obtain the statement.

(2) Let $x^{1}:=H_{1}(x), y^{1}:=H_{1}(y)$. We want to consider the winding number. To be able to do that we assume that $\infty$ is contained in the interior of the black 0-tile, furthermore we assume that $H$ deforms $\infty$ neither to $\mathcal{C}^{1}$ nor to $x^{1}$ or $y^{1}$. Then we can define the winding number $N_{\mathcal{C}}(x), N_{\mathcal{C}}(y)$ of $\mathcal{C}$ for $x$ and $y$.

Then $N_{\mathcal{C}}(x), N_{\mathcal{C}}(y)$ agree if and only if $X^{0}, Y^{0}$ have the same color. This happens if and only if $N_{\mathcal{C}^{1}}\left(x^{1}\right), N_{\mathcal{C}^{1}}\left(y^{1}\right)$ agree, these winding numbers are computed by mapping $\widehat{\mathbb{C}} \backslash\left\{H_{1}(\infty)\right\}$ (orientation preserving) to $\mathbb{C}$, i.e., identifying $\infty^{1}:=H_{1}(\infty)$ with $\infty$. The curve $\mathcal{C}^{1}=H_{1}(\mathcal{C})$ is traversed in the direction induced by $H_{1}$ as well as the orientation of $\mathcal{C}$.

Assume that $H_{1}(\infty)$ is contained in a black 1-tile. Each 1-edge is contained in exactly one white 1-tile. Consider the 1-edges $E_{1}, \ldots, E_{k}$ contained in the boundary of a white 1-tile $Z$. By (11) all these 1-edges are either positively or negatively oriented as boundary of $Z$. In the first case the winding number of $E_{1} \cup \cdots \cup E_{k}$ is 1 , in the second case -1 , for all points in the interior of $Z$. For all points in the complement of $Z$ the winding number is 0 . The same argument applies to all white 1-tiles. Note that by (10) the boundaries of all white 1-tiles have the same orientation induced by $H_{1}$, i.e., are either all positively or all negatively oriented. Since taking the union of all 1-edges in the boundaries of all white 1-tiles yields all 1-edges, it follows that the winding number of $\mathcal{C}^{1}$ for all points in the interior of some white 1 -tile is either 1 or -1 , while the winding number for all points in the interior of black 1-tiles is 0 . This finishes the claim. The argument in the case when $H_{1}(\infty)$ is contained in a white 1-tiles is completely analogous.

(3) We use the setting as above. Assume $X^{0}$ is white, then the winding number of $\mathcal{C}^{1}$ for $x^{1}$ is 1 if and only if the orientation induced by $H_{1}$ of the 1-edges in the 
boundary of $X^{1}$ agrees with the orientation of them as boundary of $X^{1}$, this happens if and only if $H$ is orientation-preserving by (11). If $X^{0}$ is white the argument is completely analogous.

\section{Connections}

There is an equivalent way to describe the existence of a pseudo-equator as in Definition 6.1. Intuitively the description is most easily explained along a picture as in Figure 2,

Namely consider the left picture in Figure 2, Each of the critical points $c_{j}$ is contained in exactly 3 white, as well as 3 black 1-tiles. Consider first the critical point $c_{0}$. Here all white 1-tiles are connected at $c_{0}$, while none of the black 1-tiles are connected at $c_{0}$.

At the critical point $c_{1}$ there are two white 1-tiles connected, and two black 1-tiles that are connected. Also there is one white, as well as one black 1-tile that is not connected to any other 1 -tile at $c_{1}$.

At the critical point $c_{2}$ all three black 1-tiles containing $c_{2}$ are connected, all three white 1-tiles containing $c_{2}$ are not connected to any other 1-tile.

The connection of white 1 -tiles at each critical point $c_{j}$ is complementary to the connection of black 1-tiles at $c_{j}$. The white, as well as the black 1-tiles, are connected in such a way that the resulting white connection graph is a spanning tree.

The connection of white 1-tiles may be represented geometrically as in Figure 2 Taking the boundary of this geometric representation of the white cluster results in a curve that is isotopic to $\mathcal{C}$ rel. post $(f)$.

We now proceed to make the above precise.

Let $X_{0}, \ldots X_{2 n-1}$ be the 1-tiles intersecting in a 1-vertex $v$, ordered mathematically positively around $v$. The white 1-tiles have even index, the black ones odd index. We consider a decomposition $\pi_{\mathrm{w}}=\pi_{\mathrm{w}}(v)$ of $\{0,2, \ldots, 2 n-2\}$ (i.e., of indices corresponding to white 1-tiles around $v)$; and a decomposition $\pi_{\mathrm{b}}=\pi_{\mathrm{b}}(v)$ of $\{1,3, \ldots, 2 n-1\}$ (i.e., of indices corresponding to black 1-tiles around $v$ ). They satisfy the following:

- They are decompositions. This means $\pi_{\mathrm{w}}=\left\{b_{1}, \ldots, b_{N}\right\}$, where each block $b_{i}$ is a subset of $\{0,2, \ldots, 2 n-2\}, b_{i} \cap b_{j}=\emptyset(i \neq j)$, and $\bigcup b_{i}=$ $\{0,2, \ldots, 2 n-2\}$. Similarly for $\pi_{\mathrm{b}}$.

- The decompositions $\pi_{\mathrm{w}}, \pi_{\mathrm{b}}$ are non-crossing. This means the following. Two distinct blocks $b_{i}, b_{j} \in \pi_{\mathrm{w}}$ are crossing if there are numbers $a, c \in b_{i}$, $b, d \in b_{j}$ and

$$
a<b<c<d .
$$

Each partition $\pi_{\mathrm{w}}, \pi_{\mathrm{b}}$ does not contain any (pair of) crossing blocks.

- The partitions $\pi_{\mathrm{w}}, \pi_{\mathrm{b}}$ are complementary. This means the following. Given $\pi_{\mathrm{w}}$, the partition $\pi_{\mathrm{b}}$ is the unique, biggest partition (of $\{1,3, \ldots, 2 n-1\}$ ) such that $\pi_{\mathrm{w}} \cup \pi_{\mathrm{b}}$ is a non-crossing partition of $\{0,1, \ldots, 2 n-1\}$.

A partition $\pi_{\mathrm{w}} \cup \pi_{\mathrm{b}}$ as above is called a complementary non-crossing partition, or cnc-partition. We may represent a cnc-partition geometrically as follows. Let $i, j \in$ $b \in \pi_{\mathrm{w}}$. We call these indices succeeding (in $\left.b\right)$, if $i+1, i+2, \ldots, j-1(\bmod 2 n) \notin b$. Let $e_{k}:=\exp (2 \pi k i / 2 n), k=0, \ldots, 2 n-1$ be the $2 n$ unit roots. Consider the closed 

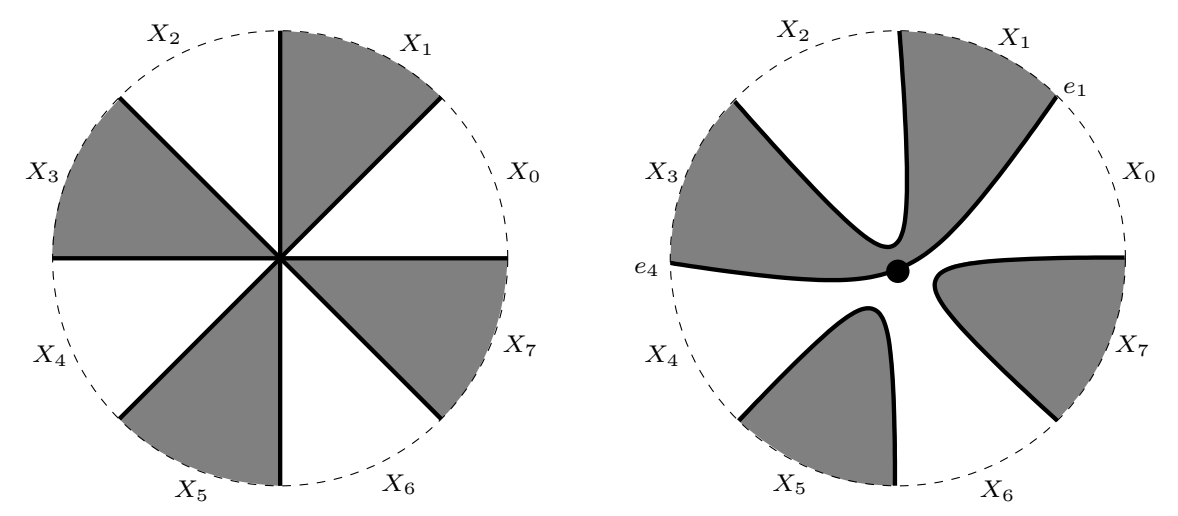

Figure 4. Connection at a vertex.

unit disk $\overline{\mathbb{D}}$. For each pair of succeeding indices $i, j$ in any white block $b \in \pi_{\text {w }}$ we draw a Jordan $\operatorname{arc} g_{m}(m=1, \ldots, n)$ in $\overline{\mathbb{D}}$ connecting $e_{i+1}, e_{j}$. Each such arc intersects $\partial \mathbb{D}$ only in its endpoints (i.e., in $e_{i+1}, e_{j}$ ). Furthermore two distinct such arcs are disjoint. Figure 4 shows a geometric representation of the cnc-partition $\pi_{\mathrm{w}} \cup \pi_{\mathrm{b}}$ given by $\pi_{\mathrm{w}}=\{\{0,4,6\},\{2\}\}, \pi_{\mathrm{b}}=\{\{1,3\},\{5\},\{7\}\}$.

The $\operatorname{arcs} g_{m}$ divide $\overline{\mathbb{D}}$ into $n+1$ components. We color those components in a checkerboard fashion black and white, such that components which share an arc $g_{m}$ as its boundary have different color. Furthermore we color the component having the circular arc between $e_{0}, e_{1}$ on $\partial \mathbb{D}$ white.

A geometric representation of a cnc-partition is to be thought of as a blow-up of the 1-edges incident to a 1-vertex $v$. We will sometimes require to know where in the geometric representation the original vertex is located. This is achieved by marking the cnc-partition. This means we mark one of the arcs $g_{m}$ above. Equivalently we may mark a pair of succeeding indices $i, j$ contained in some white block $b \in \pi_{\mathrm{w}}$. If a cnc-partition is marked we always let the marked arc $g_{m}$ contain the origin.

In Figure 2 the arc connecting $e_{0+1}, e_{4}$, equivalently the succeeding indices 0,4 of the block $\{0,4,6\}$, is marked.

A connection (of 1-tiles) assigns to each 1-vertex $v$ a cnc-partition $\pi_{\mathrm{w}}(v) \cup \pi_{\mathrm{b}}(v)$ as above. Furthermore in the case when $v=p$ is a postcritical point, the cnc-partition is marked. This marking is mostly relevant in the case when the postcritical point is at the same time a critical point, since then there are several choices which arc to mark. Two 1-tiles $X_{i}, X_{j} \ni v$ are said to be connected at $v$ if the indices $i, j$ are contained in the same block of $\pi_{\mathrm{w}}(v) \cup \pi_{\mathrm{b}}(v)$. Note that tiles of different color are never connected. The 1-tile $X_{i}$ is incident (at $v$ ) to the block $b \ni i$ of $\pi_{\mathrm{w}}(v) \cup \pi_{\mathrm{b}}(v)$.

The white connection graph is defined as follows. For each white 1-tile there is a vertex and for each block $b \in \pi_{\mathrm{w}}(v)$ (for any 1-vertex $v$ ) there is a vertex. There is an edge in the white connections graph between $b \in \pi_{\mathrm{w}}(v)$ and the white 1-tile $X \ni v$ if and only if $X$ is incident to $b$ at $v$.

A geometric representation of a connection is achieved as follows. For each 1vertex $v$ a neighborhood of $v$ "looks like" the picture on the left in Figure 4 . This is replaced by the picture on the right in Figure 4 , i.e., by a geometric representation of the cnc-partition that represents the connection at $v$. More precisely there is a neighborhood $U_{v}$ of $v$ and a homeomorphism $\varphi=\varphi_{v}: U_{v} \rightarrow \mathbb{D}$ with the following 
properties. The point $v$ is mapped to the origin. For any 1-edge $E \ni v$, the map $\varphi$ maps $E \cap U_{v}$ to some ray $R_{k}=\{r \exp (2 \pi k i / 2 n) \mid 0 \leq r<1\}$, where $k=0, \ldots, 2 n-$ 1. If $X \ni v$ is a white 1-tile, then $X \cap U_{v}$ is mapped by $\varphi$ to the sector between two rays $R_{2 k}, R_{2 k+1}$. Finally distinct 1 -vertices $v, w$ have disjoint neighborhoods $U_{v}, U_{w}$. Draw a geometric representation of the cnc-partition $\pi_{\mathrm{w}}(v) \cup \pi_{\mathrm{b}}(v)$ in $\mathbb{D}$ as above. Replace $U_{v}$ by the preimage (of this geometric representation) by $\varphi_{v}$ (for each 1-vertex $v$ ).

Given a geometric representation of a connection we obtain several black and white components. The following is Mey11, Lemma 6.23].

Lemma 7.1. Assume a connection (of 1-tiles) is given. Then the following are equivalent.

- The white connection graph is a spanning tree.

- In each geometric representation (of the connection) there is a single white component $V_{\mathrm{w}}$, which is a Jordan domain.

The following is Mey11, Lemma 7.2].

Lemma 7.2. Let $f: \widehat{\mathbb{C}} \rightarrow \widehat{\mathbb{C}}$ be a postcritically finite rational map with Julia set $\mathcal{J}(f)=\widehat{\mathbb{C}}$ and $\mathcal{C} \subset \widehat{\mathbb{C}}$ be a Jordan curve with post $(f) \subset \mathcal{C}$. Then

- there is a pseudo-isotopy $H: \widehat{\mathbb{C}} \times[0,1] \rightarrow \widehat{\mathbb{C}}$ as in Definition 6.1 (i.e., $f$ has a pseudo-equator) if and only if

- there is a connection of 1-tiles, such that the white connection graph is a spanning tree and the boundary $\partial V_{\mathrm{w}}$ (of the white component $V_{\mathrm{w}}$ from Lemma 7.1] is orientation-preserving isotopic to $\mathcal{C}$ rel. $\operatorname{post}(f)$.

Here $\partial V_{\mathrm{w}}$ is positively oriented as boundary of the white component $V_{\mathrm{w}}$ (and $\mathcal{C}$ is positively oriented as boundary of the white 0 -tile $X_{\mathrm{w}}^{0}$ as before).

Theorem 7.3. Let $f: \widehat{\mathbb{C}} \rightarrow \widehat{\mathbb{C}}$ be a postcritically finite rational map, with Julia set $\mathcal{J}(f)=\widehat{\mathbb{C}}$ and $\# \operatorname{post}(f)=3$. Then $f$ or $f^{2}$ arises as a mating.

Proof. Let $f: \widehat{\mathbb{C}} \rightarrow \widehat{\mathbb{C}}$ be as in the statement. Let $\mathcal{C} \subset \widehat{\mathbb{C}}$ be a Jordan curve with post $(f) \subset \mathcal{C}$. We choose the closure of one component of $\widehat{\mathbb{C}} \backslash \mathcal{C}$ to be $X_{\mathrm{w}}^{0}$. Then $\mathcal{C}$ is positively oriented as boundary of the white 0 -tile $X_{\mathrm{w}}^{0}$. It is easy to construct a connection of white 1-tiles such that the resulting white connection graph is a spanning tree, see Mey11, Corollary 6.20]. Indeed one starts with a connection where no white 1-tiles are connected at any 1-vertex, successively one "adds" other 1-tiles, until one obtains a spanning tree as desired.

From now on the connection (of white 1-tiles), which results in the white connection graph being a spanning tree, is fixed. Let $V_{\mathrm{w}}$ be the white component, according to Lemma 7.1 Let $\partial V_{\mathrm{w}}$ be its boundary, oriented positively as boundary of $V_{\mathrm{w}}$.

The following fact is well-known (a proof can however be found in BM11, Chapter 9.2]). Let $P \subset S^{2}$ be a set with $\# P \leq 3$ and $\gamma, \gamma^{\prime} \subset S^{2}$ be two Jordan curves, which both contain $P$. Then $\gamma, \gamma^{\prime}$ are isotopic rel. $P$. However if $\gamma, \gamma^{\prime} \subset S^{2}$ are oriented it is not necessarily true that $\gamma, \gamma^{\prime}$ are orientation-preserving isotopic rel. $P$.

If $\partial V_{\mathrm{w}}$ is orientation-preserving isotopic to $\mathcal{C}$ rel. post $(f)$ we are done by Lemma 7.2 and Theorem 6.3. i.e., $f$ arises as a mating. 
Assume $\partial V_{\mathrm{w}}$ is not orientation-preserving isotopic to $\mathcal{C}$ rel. post $(f)$. Then $\partial V_{\mathrm{w}}$ is orientation-reversing isotopic to $\mathcal{C}$ rel. $\operatorname{post}(f)$. We obtain an elementary, orientationreversing pseudo-isotopy $H$, that deforms $\mathcal{C}$ to $\mathcal{C}^{1}$ as in Lemma 6.7 (11).

Let $\widetilde{H}: \widehat{\mathbb{C}} \times[0,1] \rightarrow \widehat{\mathbb{C}}$ be the lift of $H$ by $f$ with $\widetilde{H}_{0}=\mathrm{id}_{\widehat{\mathbb{C}}}$. We note some properties of the lift $\widetilde{H}$, proofs may be found in [Mey11, Lemma 3.5 and Lemma 3.6]. Namely $\widetilde{H}$ is a pseudo-isotopy rel. $\mathbf{V}^{1}$ (= set of 1-vertices) such that $f \circ \widetilde{H}(x, t)=$ $H(f(x), t)$ for all $x \in \widehat{\mathbb{C}}, t \in[0,1]$. It deforms $\mathcal{C}^{1}$ to $\mathcal{C}^{2}:=f^{-1}\left(\mathcal{C}^{1}\right)=f^{-2}(\mathcal{C})$, i.e., $\widetilde{H}_{1}\left(\mathcal{C}^{1}\right)=\mathcal{C}^{2}$. We call $\mathbf{V}^{2}:=f^{-2}(\operatorname{post}(f))$ the set of 2 -vertices, the closure of one component of $\mathcal{C}^{2} \backslash \mathbf{V}^{2}$ is called a 2-edge. Only finitely many points $x \in \mathcal{C}^{1}$ are deformed to any 2 -vertex (i.e., to any point $\left.v \in f^{-2}(\operatorname{post}(f))\right)$. The points $x$ as before divide $\mathcal{C}^{1}$ into closed arcs. There is a bijection between such closed arcs $\widetilde{A} \subset \mathcal{C}^{1}$ and 2-edges. Finally for each such arc $\widetilde{A} \subset \mathcal{C}^{1}$ there is a 1-edge $E$ and the map $\widetilde{H}_{1}: \widetilde{A} \rightarrow E$ is a homeomorphism.

Claim. $\widetilde{H}$ deforms $\mathcal{C}^{1}$ to $\mathcal{C}^{2}$ in an orientation-reversing way.

As before this means that the orientations induced on $\mathcal{C}^{2}$ by $f: \mathcal{C}^{1} \rightarrow \mathcal{C}^{2}$ and $\widetilde{H}_{1}: \mathcal{C}^{1} \rightarrow \mathcal{C}^{2}$ disagree.

Each 2-edge $\widetilde{E}$ is mapped by $f$ homeomorphically to a 1-edge $E \subset \mathcal{C}^{1}$ (see BM11, Chapter 5.3]). Recall from Definition 6.1 (2) that there is a closed arc $A \subset \mathcal{C}$ that

is deformed by $H$ to $E$. Since $\widetilde{H}$ is the lift of $H$ by $f$, there is an $\operatorname{arc} \widetilde{A} \subset \mathcal{C}^{1}$ that is mapped homeomorphically to $\widetilde{E}$ by $\widetilde{H}_{1}$, and $f(\widetilde{A})=A$. Note that $f: \widetilde{A} \rightarrow A$, as well as $f:: \widetilde{E} \rightarrow E$, is orientation-preserving. Thus it follows that $H$ is orientationreversing if and only if $\widetilde{H}$ is orientation-reversing, proving the claim. The argument is worked out in more detail in Mey11, Lemma 3.12].

Consider $K: \widehat{\mathbb{C}} \times[0,1] \rightarrow \widehat{\mathbb{C}}$ given by $K(x, t):=\widetilde{H}(H(x, t), t)$. This is a pseudoisotopy rel. post $(f)$ that deforms $\mathcal{C}$ elementary, orientation-preserving to $\mathcal{C}^{2}=$ $f^{-2}(\mathcal{C})$. Thus it follows from Theorem 6.3 that $f^{2}$ arises as a mating.

Remark 7.4. We do not know whether it is necessary to take the second iterate $f^{2}$ in the previous theorem.

\section{CRitical PORTRAits}

In the next section we will describe an algorithm to unmate a rational map $f$. More precisely, from a pseudo-equator as in Definition 6.1 we can recover the white and black polynomial $P_{\mathrm{w}}, P_{\mathrm{b}}$ that yield $f$ as their mating. We need however a description of polynomials that is adapted to the situation. Namely we need a description in terms of external angles.

Fix an integer $d \geq 2$ ( $d$ will be the degree of the rational map $f$ as well as the polynomials $\left.P_{\mathrm{w}}, P_{\mathrm{b}}\right)$. The map $\mu: \mathbb{R} / \mathbb{Z} \rightarrow \mathbb{R} / \mathbb{Z}$ is $\mu(t):=d t \bmod 1$. We will somehow abuse notation by identifying a point $x \in \mathbb{R}$ with the corresponding equivalence class $[x] \in \mathbb{R} / \mathbb{Z}$, similarly we identify $q \in \mathbb{Q}$ with the corresponding $[q] \in \mathbb{Q}$.

Definition 8.1. A list $\mathcal{A}=A_{1}, \ldots, A_{m}$ is called a critical portrait if conditions (CP 1) $-(\mathrm{CP}$ 7) are satisfied.

$(\mathrm{CP} 1)$ Each $A_{j} \subset \mathbb{Q} / \mathbb{Z} \subset \mathbb{R} / \mathbb{Z}$ is a finite set, distinct sets $A_{i}, A_{j}$ are disjoint; 
(CP 2) $\mu$ maps each set $A_{j}$ to a single point,

$$
\mu\left(A_{j}\right)=\left\{a_{j}\right\},
$$

for all $j=1, \ldots, m$;

(CP 3) $\sum_{j}\left(\# A_{j}-1\right)=d-1$.

(CP 4) The sets are non-crossing. This means the following. Two distinct sets $A_{i}, A_{j}$ are called crossing, if there are (representatives) $s, u \in A_{i}, t, v \in A_{j}$ such that $0 \leq s<t<u<v \leq 1$, otherwise non-crossing. All distinct sets $A_{i}, A_{j}$ of the critical portrait are non-crossing.

(CP 5) No $a \in A_{j}$ is periodic under $\mu$ (for any $j=0, \ldots, m-1$ ).

The set $\mathbf{A}:=\bigcup\left\{\mu^{k}\left(A_{j}\right) \mid j=1, \ldots, m, k \geq 1\right\}$, i.e., the union of forward orbit of all angles in any of the sets $A_{j}$, then is a finite set.

(CP 6) No set $A_{j}$ contains more than one point of $\mathbf{A}$.

The following definition is somewhat technical. The reader should think of $\mathbb{R} / \mathbb{Z}=$ $S^{1}$ as being the boundary of the unit disk $\mathbb{D}$. We form the convex hull of each set $A_{j}$ (with respect to the hyperbolic metric on $\mathbb{D}$. We remove all these hulls. The closure of one remaining component is called a 1-gap. Points in the same gap should be thought of as being not separated by the sets $A_{j}$. We are really only interested in points on the unit circle.

Here is the formal definition. The points $\bigcup A_{j}$ divide the circle into closed intervals $[a, b]$, i.e., $a, b \in \bigcup A_{j}$ and $(a, b)$ does not contain any point from $\bigcup A_{j}$. A 1-gap is a union of such intervals. Two intervals $\left[a_{1}, b_{1}\right],\left[a_{2}, b_{2}\right]$ belong to the same 1-gap if and only if for any two points $c_{1} \in\left(a_{1}, b_{1}\right), c_{2} \in\left(a_{2}, b_{2}\right)$, the sets $\left\{c_{1}, c_{2}\right\}, A_{j}$ are non-crossing for all $j=1, \ldots, n$.

Two points $x, y \in \mathbb{R} / \mathbb{Z}$ belong to the same $n$-gap $(n \geq 1)$ if $\mu^{k}(x), \mu^{k}(y)$ belong to the same 1-gap for all $k=0, \ldots, n-1$.

(CP 7) There is a constant $n_{0} \in \mathbb{N}$ such that the following holds. Two distinct points $a, b \in \mathbf{A}$ are not contained in the same $n_{0}$-gap.

Theorem 8.2 (Bielefeld-Fisher-Hubbard BFH92). Let $\mathcal{A}$ be a critical portrait as in Definition 8.1. Then there is a (unique up to affine conjugacy) monic polynomial $P$ of degree $d$ realizing it. The polynomial $P$ is postcritically finite, each critical point of $P$ is strictly preperiodic.

That a polynomial $P$ realizes a critical portrait means that for each $A_{j}$ there is a critical point $c_{j}$ of $P$. The degree of $P$ at $c_{j}$ is $\# A_{j}$. If $a \in A_{j}$ then the external ray $R(a)$ lands at $c_{j}$. There is a generalization of the above theorem due to Poirier Poi09].

\section{UNMATING THE MAP}

A pseudo-equator for $f$ as in Definition 6.1 does not only guarantee that $f$ arises as a mating, but it is possible to explicitly find the polynomials that when mating give the map $f$. This is described here.

Let $\mathcal{C} \supset \operatorname{post}(f)$ be a pseudo-equator for the (rational, postcritically finite) map $f: \widehat{\mathbb{C}} \rightarrow \widehat{\mathbb{C}}$, whose Julia set is the whole sphere.

Let $X_{w}^{0}, X_{b}^{0}$ be the two 0-tiles (defined in terms of $\mathcal{C}$ ) which are colored white and black. We orient $\mathcal{C}$, so that it is positively oriented as boundary of (the white 0-tile) $X_{\mathrm{w}}^{0}$. 
Recall that the postcritical points divide $\mathcal{C}$ into (closed) 0-edges $E_{0}, \ldots, E_{k-1}$, which we label positively on $\mathcal{C}$.

Consider now a 1-edge $E^{1}$. We say it is of type $j$ if $f\left(E^{1}\right)=E_{j}$. Each 0-edge is deformed by $H_{1}$ into several 1-edges. We record how many 1-edges of each type are contained in such a deformed 0-edge in the matrix $M=\left(m_{i j}\right)$ defined as follows:

$$
m_{i j}:=\text { number of 1-edges of type } j \text { contained in } H_{1}\left(E_{i}\right) .
$$

This matrix is called the edge replacement matrix of the pseudo-isotopy $H$. Since there are exactly $n$-edges of each type it follows that $\sum_{i} m_{i j}=d=\operatorname{deg} f$. It is relatively easy to show that the matrix $M$ is primitive, i.e., that $M^{n}>0$ for some $n \in \mathbb{N}$. Thus it follows from the Perron-Frobenius theorem that $d$ is a simple eigenvalue (which is in fact the spectral radius of $M$ ), with eigenvector $l=\left(l_{j}\right)>0$. We normalize $l$ by $\sum l_{j}=1$, this makes $l$ unique.

To illustrate we consider the pseudo-isotopy shown in Figure 2, Here the edge replacement matrix and the corresponding eigenvector is

$$
M=\left(\begin{array}{lll}
2 & 2 & 1 \\
2 & 1 & 2 \\
0 & 1 & 1
\end{array}\right), \quad l=\frac{1}{15}\left(\begin{array}{l}
7 \\
6 \\
2
\end{array}\right) .
$$

The vector $l$ describes the lengths of the 0 -edges. This in turn will be used to find the external angles at the 0 -vertices (= postcritical points). More precisely for each $p \in \operatorname{post}(f)$ we will define an external angle $\theta(p)$.

The 0 -edges $E_{j} \subset \mathcal{C}$ inherit the orientation of $\mathcal{C}$. Let $p_{0}, \ldots, p_{k-1}$ be the postcritical points, labeled in positively cyclical order on $\mathcal{C}$, such that $p_{0}$ is the initial point of (the first) 0-edges $E_{0}$.

Assume first that $p_{0}$ is a fixed point of $f$ (as in the example from Figure 2). Then we set the external angle of $p_{0}$ equal to 0, i.e., $\theta\left(p_{0}\right)=0$. The external angle of the other postcritical points is now given by $\theta\left(p_{1}\right)=l_{0}, \theta\left(p_{2}\right)=l_{0}+l_{1}, \ldots, \theta\left(p_{j}\right)=$ $l_{0}+\cdots+l_{j-1}$. Thus the difference between the external angles of $p_{j}$ and $p_{j+1}$ is always given by $l_{j}$ (here indices are taken $\bmod k$ ).

Assume now that $p_{0}$ is mapped by $f$ to $p_{j}$. Let $l\left(p_{0}, p_{j}\right):=l_{0}+\cdots+l_{j-1}$, i.e., the total length of all 0 -edges between $p_{0}$ and $p_{j}$ (in the positive direction on $\mathcal{C}$ ). We now desire that $d \theta\left(p_{0}\right)=\theta\left(p_{j}\right)=\theta\left(p_{0}\right)+l\left(p_{0}, p_{j}\right)$. Thus we define

$$
\theta\left(p_{0}\right)=\frac{l\left(p_{0}, p_{j}\right)}{d-1}
$$

and $\theta\left(p_{1}\right)=\theta\left(p_{0}\right)+l_{0}, \ldots, \theta\left(p_{i}\right)=\theta\left(p_{0}\right)+l_{0}+\cdots+l_{i-1}$. We will however only need $\theta\left(p_{0}\right)$ in the following.

We now assign external angles at the critical points. More precisely we want to find the external angles at critical points of polynomials $P_{\mathrm{w}}, P_{\mathrm{b}}$ (called the white/black polynomials). These are the polynomials into which $f$ is unmated, i.e., $f$ will be (topologically equivalent to) the mating of $P_{\mathrm{w}}, P_{\mathrm{b}}$. The external angles will give the critical portraits of the polynomials $P_{\mathrm{w}}, P_{\mathrm{b}}$.

First we define the length of a 1-edge $E^{1}$ of type $j$ by $l\left(E^{1}\right)=l_{j} / d$. Note that there are $d 1$-edges of each type, thus $\sum l\left(E^{1}\right)=1$ (where the sum is taken over all 1-edges).

Denote by $\mathbf{E}^{1}$ the set of all 1-edges. Since $H$ deforms $\mathcal{C}$ to $\mathcal{C}^{1}=\bigcup \mathbf{E}^{1}$ it follows that the orientation of $\mathcal{C}$ together with $H$ induces a cyclical ordering $E_{0}^{1}, \ldots, E_{k d-1}^{1}$ on the 1-edges, as well as an orientation on each 1-edge. Here we start the labeling 
at $p_{0}$, i.e., the initial point of $E_{0}^{1}$ is $p_{0}$. Note that the type of 1-edges in this cyclical ordering is changing cyclically, i.e., if $E_{i}^{1}$ is of type $j$, then $E_{i+1}^{1}$ is of type $j+1$ (here the lower index is taken $\bmod k d$, the type is taken $\bmod k$ ).

Assume the 1-edge $E_{j}^{1}$ ends at the critical point $c$. Then an external angle associated with $c$ is

$$
\theta\left(E_{j}^{1}\right):=\theta\left(p_{0}\right)+l\left(E_{0}^{1}\right)+\cdots+l\left(E_{j}^{1}\right) .
$$

There $\operatorname{are~} \operatorname{deg}_{f}(c)$ such (oriented) 1-edges ending at $c$, hence different external angles associated to $c$. We will have to decide, which belong to critical points of the white polynomial, and which belong to critical points of the black polynomial. However, the situation is more complicated: $c$ might be associated to several distinct critical points of the white polynomial, as well as several distinct critical points of the black polynomial.

Recall that the set $\mathbf{W}$ was the set of preimages of the 1-vertices by $H_{1}$ located on $\mathcal{C}$ (see Definition 6.1(2)). The points in $\mathbf{W}$ divide $\mathcal{C}$ into closed arcs, each of which is mapped by $H_{1}$ homeomorphically to a 1-edge by $H_{1}$ (see Definition 6.1 (3) ). Since there are as many such arcs as 1-edges (i.e., $k d$ ), there are $k d$ points in $\mathbf{W}$. Let the points in $\mathbf{W}$ be $w_{0}, \ldots, w_{k d-1}$ labeled positively on $\mathcal{C}$, such that $w_{0}=p_{0}$.

Consider $[c]:=\left(H_{1}\right)^{-1}(c)$, i.e., the set of points that are deformed by $H$ to $c$. This set contains $\operatorname{deg}_{f}(c)$ points of $\mathbf{W}=\left\{w_{j}\right\} \subset \mathcal{C}$ (see Remark 6.2). Recall from Lemma 2.4 that this set is connected. To clarify: the set of points in $\mathcal{C}$ that is deformed by $H$ to $c$ (i.e., $[c] \cap \mathcal{C}$ ) is finite, while the set of all points in $\widehat{\mathbb{C}}$ that is deformed by $H$ to $c$ (i.e., $[c]$ ) is infinite.

Consider the components of $[c] \backslash \mathcal{C}$. Such a component $C$ is called white/black if it is contained in the white/black 0-tile (i.e., in $X_{\mathrm{w}}^{0}$ or $X_{\mathrm{b}}^{0}$ ). Furthermore we call $C$ non-trivial if it contains at least two distinct points $w_{i}, w_{j} \in \mathbf{W}$ in its boundary. Each non-trivial white component $C$ corresponds to a critical point of the white polynomial, each non-trivial black component $C$ corresponds to a critical point of the black polynomial.

Let $C$ be a white non-trivial component. The set of external angles associated to $C$ is now the set

$$
\left\{\theta\left(E_{j}^{1}\right) \mid w_{j} \in \mathbf{W} \text { contained in the closure of } C\right\} .
$$

The list of all these sets of external angles (for all critical points $c$ and all white components of $[c] \backslash \mathcal{C}$ ) forms the critical portrait of the white polynomial $P_{\mathrm{w}}$.

The white critical portrait for the pseudo-equator indicated in Figure 2 is

$$
\left\{\frac{7}{60}, \frac{22}{60}, \frac{37}{60}\right\},\left\{\frac{43}{60}, \frac{58}{60}\right\} \text {. }
$$

The critical portrait of the black polynomial is constructed in almost the same fashion. There is a slight difference however. This difference appears, since in the construction of mating a point with external angle $\theta$ in the Julia set of one polynomial is identified with a point with external angle $-\theta$ from the Julia set of the other polynomial.

Thus let $C$ be a non-trivial black component of $[c] \backslash \mathcal{C}$ (for some critical point $c$ ). Then the set of external angles associated to $C$ is

$$
\left\{1-\theta\left(E_{j}^{1}\right) \mid w_{j} \in \mathbf{W} \text { contained in the closure of } C\right\} .
$$


The list of all these sets (for all critical points $c$ and all non-trivial black components of $[c] \backslash \mathcal{C}$ ) form the critical portrait of the black polynomial $P_{\mathrm{b}}$.

The black critical portrait of the pseudo-equator in Figure 2 is

$$
\left\{\frac{15}{60}, \frac{30}{60}, \frac{45}{60}\right\},\left\{\frac{2}{60}, \frac{47}{60}\right\} \text {. }
$$

Thus the critical portraits of the white and black polynomials can be read off from the pseudo-isotopy in an elementary combinatorial way. These determine the white and black polynomials $P_{\mathrm{w}}, P_{\mathrm{b}}$ uniquely. In Mey11 and Mey it is shown that $P_{\mathrm{w}} \Perp P_{\mathrm{b}}$ is topologically conjugate to $f$. Thus the existence of a pseudo-equator not only shows that $f$ arises as a mating, but allows to recover the polynomials into which $f$ unmates in an elementary fashion.

\section{EXAmples of UNMATings}

Here we show several examples of unmatings.

We first show that shared matings are ubiquitous. We list all shared matings of the example from Section 5 that can be found with the sufficient condition from Section 6]

The critical portraits of the polynomials into which the map $f$ unmates are as follows. They are obtained from the pseudo-equators shown in Figure 5. It is convenient to write the common denominator of the angles of a critical portrait outside the parentheses, i.e., we write $\frac{1}{N}\{n, m\},\{i, j\}$ for $\{n / N, m / N\},\{i / N, j / N\}$ and so on. By w we denote the critical portrait of the white polynomial, by $\mathrm{b}$ the critical portrait of the (corresponding) black polynomial into which $f$ unmates.

$$
\begin{array}{ll}
\text { w }: \frac{1}{60}\{7,22,37\},\{43,58\} & \text { b }: \frac{1}{60}\{2,47\},\{15,30,45\} \\
\text { w }: \frac{1}{20}\{2,7,17\},\{10,15\} & \text { b : } \frac{1}{20}\{2,7,17\},\{10,15\} \\
\text { w }: \frac{1}{60}\{11,26\},\{29,59\},\{30,45\} & \text { b : } \frac{1}{60}\{1,46\},\{19,34\},\{15,45\} \\
\text { w : } \frac{1}{60}\{14,59\},\{26,41\},\{15,45\} & \text { b : } \frac{1}{60}\{1,31\},\{15,30\},\{34,49\} \\
\text { w : } \frac{1}{20}\{3,18\},\{7,17\},\{10,15\} & \text { b : } \frac{1}{20}\{3,18\},\{7,17\},\{10,15\} \\
\text { w }: \frac{1}{60}\{7,37\},\{15,30\},\{43,58\}, & \text { b : }: \frac{1}{60}\{2,47\},\{15,45\},\{23,38\}
\end{array}
$$

The pseudo-equators in Figure 5 are not all possible, all others however are obtained from these by rotation (by $2 \pi / 3$ and $4 \pi / 3$ ). In the above the point labeled $p$ in Figure 5 is the point -1 . We now rotate each pseudo-equator by $2 \pi / 3$, meaning the point labeled $p$ is 1 . From these new pseudo-equators we obtain unmatings of $f$ into polynomials with the following critical portraits. 
UNMATING OF RATIONAL MAPS, SUFFICIENT CRITERIA AND EXAMPLES 27
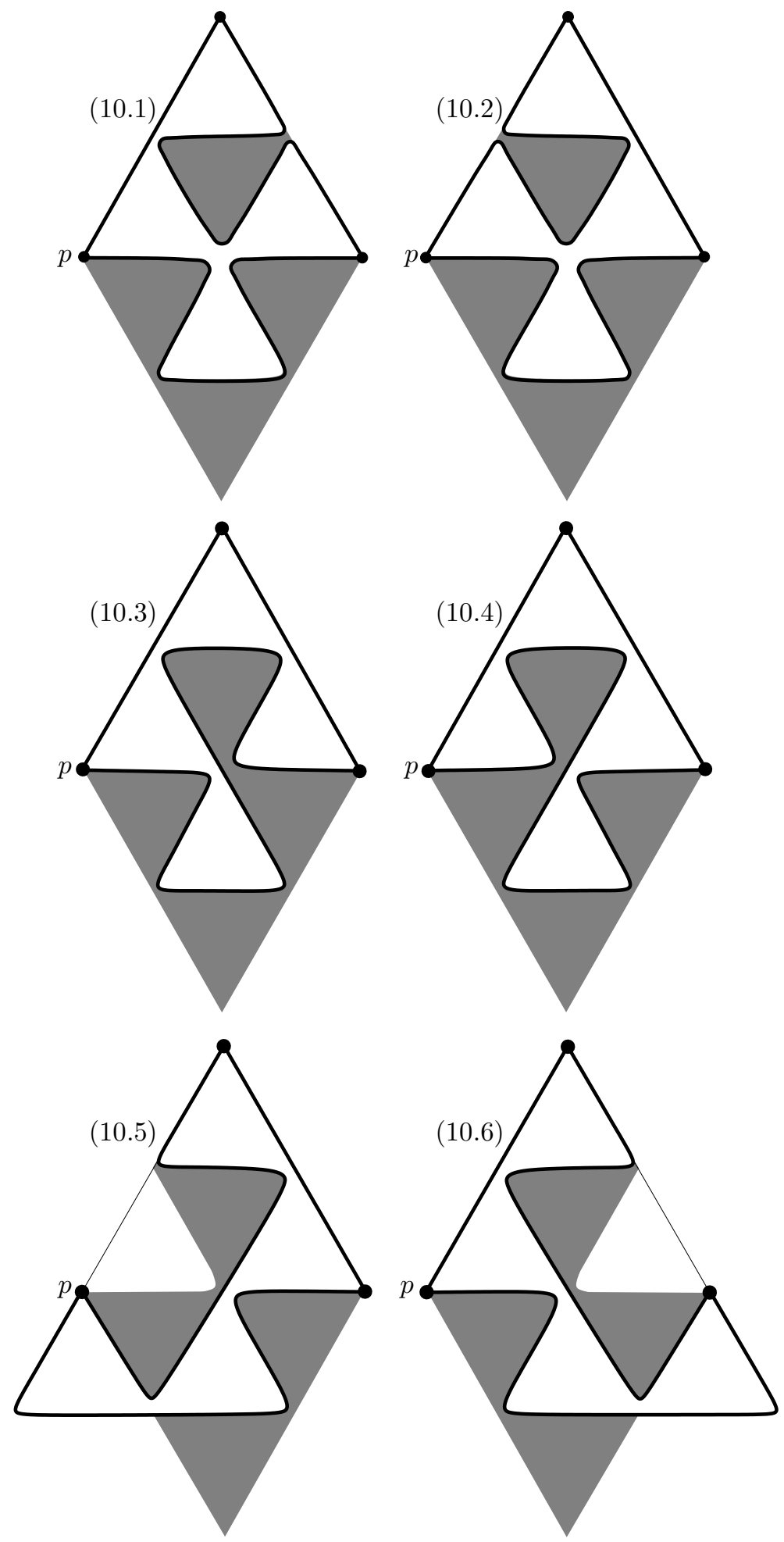

FiguRE 5. Different pseudo-equators. 


$\begin{array}{lll}\text { (10.1) } & \text { w }: \frac{1}{60}\{2,47\},\{15,30,45\} & \text { b : } \frac{1}{60}\{7,22,37\},\{43,58\} \\ \text { (10.2) } & \text { w }: \frac{1}{60}\{13,58\},\{15,30,45\} & \text { b }: \frac{1}{60}\{2,17\},\{23,38,53\} \\ \text { (10.3) } & \text { w : } \frac{1}{20}\{1,11\},\{5,10\},\{14,19\} & \text { b }: \frac{1}{20}\{1,11\},\{5,10\},\{14,19\} \\ & \text { w }: \frac{1}{20}\{1,6\},\{9,19\},\{10,15\} & \text { b }: \frac{1}{20}\{1,6\},\{9,19\},\{10,15\} \\ & \text { w }: \frac{1}{60}\{13,58\},\{15,45\},\{22,37\} & \text { b }: \frac{1}{60}\{2,17\},\{23,53\},\{30,45\} \\ & \text { w }: \frac{1}{60}\{2,47\},\{15,45\},\{23,38\} & \text { b }: \frac{1}{60}\{7,37\},\{15,30\},\{43,58\}\end{array}$

Finally we rotate the pseudo-equators in Figure 5 by $4 \pi / 3$, meaning that the point labeled $p$ is $\infty$. We obtain unmatings of the map $f$ into polynomials with the following critical portraits.

\begin{tabular}{|c|c|c|}
\hline (10.1') & $\mathrm{w}: \frac{1}{20}\{3,13,18\},\{5,10\}$ & $\mathrm{b}: \frac{1}{20}\{3,13,18\},\{5,10\}$ \\
\hline (10.2' ) & w : $\frac{1}{60}\{2,17\},\{23,38,53\}$ & $\mathrm{b}: \frac{1}{60}\{13,58\},\{15,30,45\}$ \\
\hline (10.3') & w : $\frac{1}{60}\{1,46\},\{15,45\},\{19,34\}$ & b : $\frac{1}{60}\{11,26\},\{29,59\},\{30,45\}$ \\
\hline (10.4') & w : $\frac{1}{60}\{1,31\},\{15,30\},\{34,49\}$ & $\mathrm{b}: \frac{1}{60}\{14,59\},\{15,45\},\{26,41\}$ \\
\hline & w : $\frac{1}{60}\{2,17\},\{23,53\},\{30,45\}$ & $\mathrm{b}: \frac{1}{60}\{13,58\},\{15,45\},\{22,37\}$ \\
\hline 10.6' ) & $\mathrm{w}: \frac{1}{20}\{2,17\},\{3,13\},\{5,10\}$ & $\mathrm{b}: \frac{1}{20}\{2,17\},\{3,13\},\{5,10\}$ \\
\hline
\end{tabular}

Here are some observations from these examples. Several, namely (10.2), (10.5), (10.31), (10.4]), (10.1], (10.6), are obtained by mating the same white polynomial to the same black polynomial.

Somewhat more interesting (and possibly surprising) is the following phenomenon. Consider the pairs (10.1) and (10.17), (10.6) and (10.6), (10.3) and (10.3), (10.4) and (10.4), as well as (10.2 and (10.2). In each of these cases the map $f$ is unmated into the same two polynomials. The pseudo-equators however by which these unmatings are achieved are distinct. Thus the corresponding invariant Peano curves are distinct. Put differently, $f$ can be obtained as the mating of two polynomials $P_{\mathrm{w}}, P_{\mathrm{b}}$ in two distinct ways. Note that the role of the black and white polynomials however is interchanged in each case.

We next consider some unmatings of the map $g: \widehat{\mathbb{C}} \rightarrow \widehat{\mathbb{C}}$ given by

$$
g(z)=1+\frac{\omega-1}{z^{3}}
$$

where $\omega=\exp (4 \pi i / 3)$. Note that $g(z)=\tau\left(z^{3}\right)$, where $\tau(w)=1+(\omega-1) / w$ is a Möbius transformation that maps the upper half plane to the half plane above the line through $\omega, 1$ (more precisely $\tau: 0 \mapsto \infty, 1 \mapsto \omega, \infty \mapsto 1$ ). The critical points of $g$ are $0, \infty$, which are mapped as follows. 

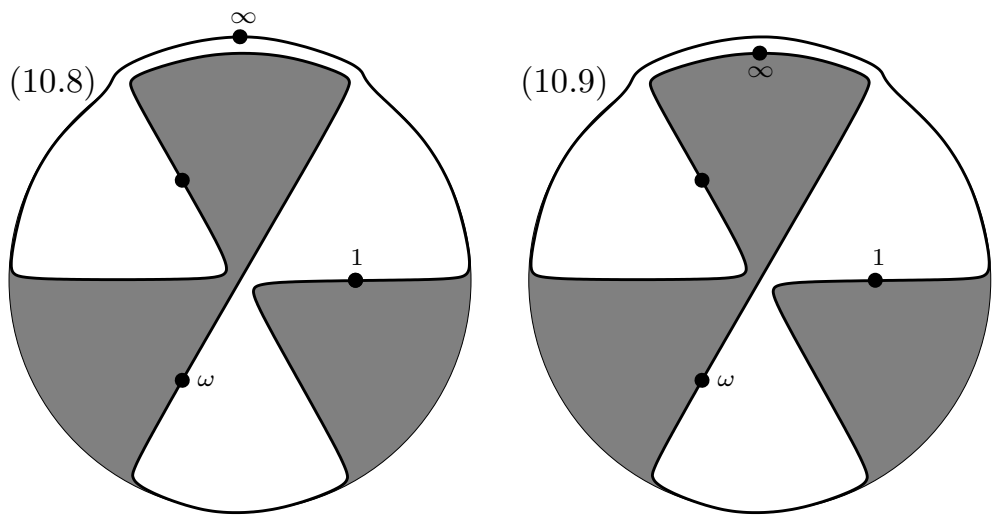

Figure 6. Shared invariant Peano curve.

$$
0 \stackrel{3: 1}{\longrightarrow} \infty \stackrel{3: 1}{\longrightarrow} 1 \longrightarrow \stackrel{\overbrace{}}{\omega}
$$

Thus $\operatorname{post}(g)=\{\infty, 1, \omega\}$, i.e., $g$ is postcritically finite. Since each critical point is strictly preperiodic, it follows that the Julia set of $g$ is all of $\widehat{\mathbb{C}}$. The crucial property for our purposes is that $\infty$ is at the same time a critical as well as a postcritical point.

As $\mathcal{C} \supset \operatorname{post}(g)$ we choose the extended line through $\omega, 1$, i.e., the circle on $\widehat{\mathbb{C}}$ through $\omega, 1, \infty$, oriented positively as boundary of the half plane above this line. It holds

$$
g^{-1}(\mathcal{C})=\bigcup_{j=0, \ldots, 5} R_{j}
$$

where $R_{j}=\{r \exp (2 \pi i j / 6) \mid 0 \leq r \leq \infty\}$.

We show two pseudo-equators for $g$ in Figure 6. In both cases the points on the circle all represent the point $\infty$. The narrow white channel indicates that two white 1-tiles are connected at $\infty$ (in both cases). The connection of 1-tiles is the same in (10.8) and (10.9), with one exception: the marking of the connection at $\infty$ (more precisely the cnc-partition representing the connection at $\infty$ ) differ. This means that in the geometric representation of Figure 6 the point $\infty$ is in different positions. From these pseudo-equators we obtain unmatings of $g$ into polynomials with the following critical portraits.

$$
\begin{array}{ll}
\text { w : } \frac{1}{27}\{4,22\},\{12,21\} & \text { b : } \frac{1}{27}\{5,14\},\{15,24\} \\
\text { w : } \frac{1}{27}\{7,25\},\{12,21\} & \text { b : } \frac{1}{27}\{2,11\},\{15,24\}
\end{array}
$$

Remark 10.1. At first the author thought that this example would result in two distinct pairs of polynomials that would do not only yield the same rational map when mated, but also the same invariant Peano curve. This however is not true. We do not give the precise argument here. This can however be found in Mey11, Section 8]. 


\section{A mating not ARISIng From a PSEudo-EQuator}

In this section we give an example of a mating that does not arise via a pseudoequator. Thus we show that the sufficient condition that a rational map arises as a mating given in Theorem 6.3 is not necessary.

More precisely we will mate two postcritically finite polynomials and show that the resulting rational map has no pseudo-equator. The Julia set of this map will be the whole sphere.

In the construction of the topological mating a point in the white Julia set where the external ray $R_{\theta}$ lands is identified with a point in the black Julia set where the external ray $R_{-\theta}$ lands. To make the following more readable we define for any $\theta \in[0,1) \theta^{*}:=1-\theta$. Thus in the topological mating the landing point of $R_{\theta}$ in the white Julia set is identified with the landing point of $R_{\theta^{*}}$ in the black polynomial.

The white polynomial will be $P_{\mathrm{w}}:=z^{2}+i$. It is well-known that the critical portrait of $P_{\mathrm{w}}$ is $\{1 / 12,7 / 12\}$. The external angles are mapped under the angle doubling map in the following way:

$$
(1 / 12),(7 / 12) \longrightarrow(1 / 6) \longrightarrow(1 / 3) \longrightarrow(2 / 3)
$$

Furthermore at the $\alpha$-fixed point of $P_{\mathrm{w}}$ the external rays with angles $\{1 / 7,2 / 7,4 / 7\}$ land.

As the black polynomial $P_{\mathrm{b}}$ we pick the (quadratic, monic, postcritically finite) polynomial with the critical portrait $\left\{(1 / 28)^{*},(15 / 28)^{*}\right\}$. Thus $P_{\mathrm{b}}=z^{2}+c_{\mathrm{b}}$, where $c_{\mathrm{b}}$ is the landing point of the external ray $R_{(1 / 14)^{*}}$ in the Mandelbrot set. The external angles are mapped under angle doubling in the following way:

$$
(1 / 28)^{*},(15 / 28)^{*} \longrightarrow(1 / 14)^{*} \longrightarrow(1 / 7)^{*} \longrightarrow(2 / 7)^{*} \longrightarrow(4 / 7)^{*} \text {. }
$$

Lemma 11.1. The topological mating of $P_{\mathrm{w}}, P_{\mathrm{b}}$ exists and is topologically conjugate to a rational map $f: \widehat{\mathbb{C}} \rightarrow \widehat{\mathbb{C}}$ of degree 2 . The two critical points a, $b$ of $f$ are mapped by $f$ in the following way:

$$
\begin{aligned}
& a \stackrel{2: 1}{\longrightarrow} p_{1} \longrightarrow p_{2} \\
& b \stackrel{2: 1}{\longrightarrow} q_{1} \longrightarrow q_{2} \longrightarrow q_{3} .
\end{aligned}
$$

Thus $f$ has 5 postcritical points, namely $p_{1}, p_{2}, q_{1}, q_{2}, q_{3}$. The Julia set of $f$ is the whole sphere, since each critical point is strictly preperiodic.

Proof. It is well-known that $i$, i.e., the critical value of $P_{\mathrm{w}}$ is in the $1 / 3$-limb of the Mandelbrot set. Indeed the external angle at the critical value $i$ is $1 / 6$. The external rays $R_{1 / 7}$ and $R_{2 / 7}$ disconnect the $1 / 3$-limb from the main cardioid. Since $1 / 7<1 / 6<2 / 7$ it follows that $i$ lies in this limb.

The conjugate limb is the $2 / 3$-limb, it is disconnected from the main cardioid by the external rays $R_{5 / 7}, R_{6 / 7}$. Since $(1 / 14)^{*}=(13 / 14)>6 / 7$, it follows that $i, c_{\mathrm{b}}$ do not lie in conjugate limbs of the Mandelbrot set. From the Rees-Shishikura-Tan theorem (Theorem 3.2) it follows that the topological mating of $P_{\mathrm{w}}, P_{\mathrm{b}}$ exists and is topologically conjugate to a rational map (which is postcritically finite and of degree 2).

Clearly the three postcritical points of $P_{\mathrm{b}}$ with external angles $(1 / 7)^{*},(2 / 7)^{*}$, $(4 / 7)^{*}$ are all identified with the $\alpha$-fixed point of $P_{\mathrm{w}}$ under the topological mating, 
i.e., correspond to a single postcritical point in the mating. It remains to show that there are not more identifications of postcritical points.

Consider first the external angle $1 / 3$. Let $z_{(1 / 3)^{*}}$ be the landing point of $R_{(1 / 3)^{*}}$ in the Julia set $\mathcal{J}_{\mathrm{b}}$ of $P_{\mathrm{b}}$. We want to show that $R_{(1 / 3)^{*}}$ is the only external ray landing at $z_{(1 / 3)^{*}}$. Indeed $(1 / 3)^{*}$ is fixed under the second iterate of the angledoubling map. Thus if $R_{\theta^{*}}$ lands at $z_{(1 / 3)^{*}}$ it has to be fixed under the second iterate of the angle doubling map as well. Thus either $\theta^{*}=(2 / 3)^{*}$ or $\theta^{*}=0$. In the first case the external rays $R_{1 / 3}, R_{2 / 3}$ in the dynamical plane of $P_{\mathrm{w}}$ together with the external rays $R_{(1 / 3)^{*}}, R_{(2 / 3)^{*}}$ disconnect the formal mating sphere. This cannot happen by the Rees-Shishikura-Tan theorem. The second case cannot happen, since for quadratic polynomials the landing point of $R_{0}$, i.e., the $\beta$-fixed point, is not the landing point of any other external ray.

By the same argument it follows that the landing point of $R_{(2 / 3)^{*}}$ (in the dynamical plane of $P_{\mathrm{b}}$ ) is not the landing point of any other external ray.

Consider now the landing point $z_{(1 / 6)^{*}}$ of $R_{(1 / 6)^{*}}$, in the dynamical plane of $P_{\mathrm{b}}$. This point is not a critical point which is mapped to $z_{(1 / 3) *}$ by $P_{\mathrm{b}}$. Since by the above there is a single external ray landing at $z_{(1 / 3)^{*}}$ it follows that there is a single external ray landing at $z_{(1 / 6)^{*}}$.

Thus it follows that the three postcritical points of $P_{\mathrm{w}}$ descend to three distinct postcritical points $q_{1}, q_{2}, q_{3}$ of $f$ and their dynamics is as described in the statement.

It remains to show that the postcritical point of $P_{\mathrm{b}}$ at which the external ray $R_{(1 / 14)^{*}}$ (in the dynamical plane of $P_{\mathrm{b}}$ ) lands is not identified with other postcritical or critical points.

We first note that at the three postcritical points of $P_{\mathrm{b}}$ at which the external rays $R_{(1 / 7)^{*}}, R_{(2 / 7)^{*}}, R_{(4 / 7)^{*}}$ land no other external ray lands. Indeed any other such external ray would be a fixed point of the third iterate of the angle doubling map, i.e., has to be an external ray of the form $R_{(k / 7)^{*}}$, where $k=3,5,6$. Assume first that $R_{(1 / 7)^{*}}, R_{(6 / 7)^{*}}$ land at the same point. This is impossible, since the external rays $R_{(1 / 28)^{*}}, R_{(15 / 28)^{*}}$ (which both land at the critical point of $P_{\mathrm{b}}$ ) are not contained in one component of $\mathbb{C} \backslash\left(R_{(1 / 7)^{*}} \cup R_{(6 / 7)^{*}}\right.$. The same argument shows that $R_{(1 / 7)^{*}}, R_{(5 / 7)^{*}}$ cannot land at the same point. Finally assume that $R_{(1 / 7)^{*}}, R_{(3 / 7)^{*}}$ land at the same point. Mapping these external rays by $P_{\mathrm{b}}$ yields that $R_{(2 / 7)^{*}}, R_{(6 / 7)^{*}}$ land at the same point. Again this is impossible, since $R_{(1 / 28)^{*}} \cup R_{(15 / 28)^{*}}$ do not lie in the same component of $\mathbb{C} \backslash\left(R_{(2 / 7)^{*}} \cup R_{(6 / 7)^{*}}\right)$.

Consider the landing point $z_{1 / 14}$ of the external ray $R_{1 / 14}$ in the dynamical plane of $P_{\mathrm{w}}$. This is the preimage of the $\alpha$-fixed point of $P_{\mathrm{w}}$. Thus the external rays $R_{9 / 14}, R_{11 / 14}$ (in the dynamical plane of $P_{\mathrm{w}}$ ) land at $z_{1 / 14}$ as well.

Consider now one the corresponding external rays $R_{(9 / 14)^{*}}, R_{(11 / 14)^{*}}$ in the dynamical plane of $R_{\mathrm{b}}$. These are mapped by $P_{\mathrm{b}}$ to $R_{(2 / 7)^{*}}, R_{(4 / 7)^{*}}$. By the above there is no other external ray landing at these endpoints. Thus there are no other external rays landing at the same points as $R_{(9 / 14)^{*}}, R_{(11 / 14)^{*}}$.

Thus the 4 postcritical points of $P_{\mathrm{b}}$ descend to 2 postcritical points $p_{1}, p_{2}$ of $f$, which are mapped as stated.

Theorem 11.2. The rational map $f: \widehat{\mathbb{C}} \rightarrow \widehat{\mathbb{C}}$ from Lemma 11.1, which arises as the mating of $P_{\mathrm{w}}$ and $P_{\mathrm{b}}$, does not have a pseudo-equator (as in Definition 6.1). 

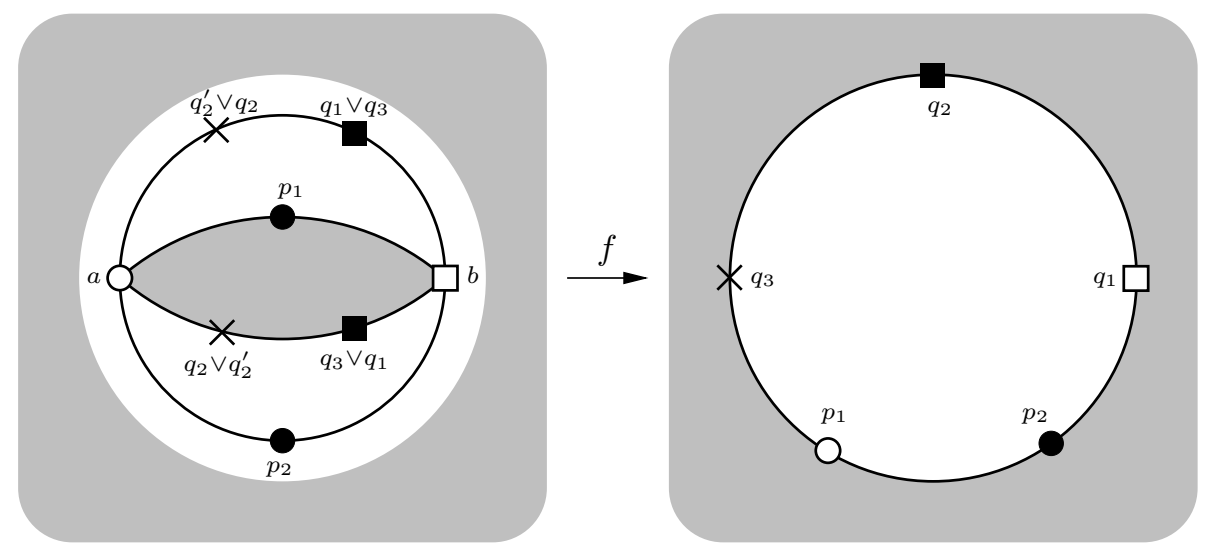

Figure 7. Case 1.

Proof. To show that no pseudo-equator as in Definition 6.1 exists, we will consider several cases. Namely we consider the different cyclical orders in which $\mathcal{C}$ may traverse the postcritical points. Assume a pseudo-isotopy $H: \widehat{\mathbb{C}} \times[0,1] \rightarrow \widehat{\mathbb{C}}$ deforms $\mathcal{C}$ to $\mathcal{C}^{1}=f^{-1}(\mathcal{C})$ as in Definition 6.1. Then $H$ induces an order in which $\mathcal{C}^{1}$ is traversed. The cyclical order in which $\mathcal{C}^{1}$ traverses the postcritical points has to agree with the cyclical order of the postcritical points on $\mathcal{C}$, since $H$ is a pseudoisotopy rel. post $(f)$. We will show that this is impossible.

Thus let $\mathcal{C} \subset \widehat{\mathbb{C}}$ be a Jordan curve with $\operatorname{post}(f) \subset \mathcal{C}$, which is (arbitrarily) oriented. The white and black 0-tiles $X_{\mathrm{w}}^{0}, X_{\mathrm{b}}^{0}$, as well as the white and black 1-tiles are defined in terms of $\mathcal{C}$ as usual. There are two white (as well as two black) 1-tiles, which intersect at the critical points $a, b$. To obtain a connection of white 1-tiles that yields a spanning tree, we have to connect the two white 1-tiles either at $a$ or at $b$. Recall that each white 1-tile is mapped homeomorphically to $X_{\mathrm{w}}^{0}$ by $f$. Thus the two preimages of any point $p_{2}, q_{2}, q_{3}$, i.e., the postcritical points which are not critical values, have to lie in distinct white 1-tiles.

Let $q_{2}^{\prime}$ be the preimage of $q_{3}$ by $f$ distinct from $q_{2}$. Augmenting this point to the diagram in Lemma 11.1 we obtain that the points $a, b, p_{1}, p_{2}, q_{1}, q_{2}, q_{2}^{\prime}, q_{3}$ are mapped by $f$ as follows:

$$
\begin{aligned}
& a \stackrel{2: 1}{\longrightarrow} p_{1} \longrightarrow p_{2} \\
& b \stackrel{2: 1}{\longrightarrow} q_{1} \longrightarrow q_{2} \longrightarrow q_{3} \longleftrightarrow q_{2}^{\prime} .
\end{aligned}
$$

Note that the set $\left\{a, b, p_{1}, p_{2}, q_{1}, q_{2}, q_{2}^{\prime}, q_{3}\right\}$ contains all preimages of the postcritical points.

Case $1\left(p_{1}, p_{2}\right.$ are adjacent on $\left.\mathcal{C}\right)$.

This means that one arc on $\mathcal{C}$ between $p_{1}, p_{2}$ does not contain any other postcritical point. One such situation is pictured in Figure 7 Assume the two white 1-tiles are connected at $b$. Traverse $\mathcal{C}^{1}:=f^{-1}(\mathcal{C})$ in the order induced by this connection. Note that on each of the two paths (on $\mathcal{C}^{1}$ ) between $p_{1}, p_{2}$ there is at least one other postcritical point. Thus there is no pseudo-isotopy rel. post $(f)$ that deforms $\mathcal{C}$ to $\mathcal{C}^{1}$. The same argument works in the case when the white 1-tiles are connected 

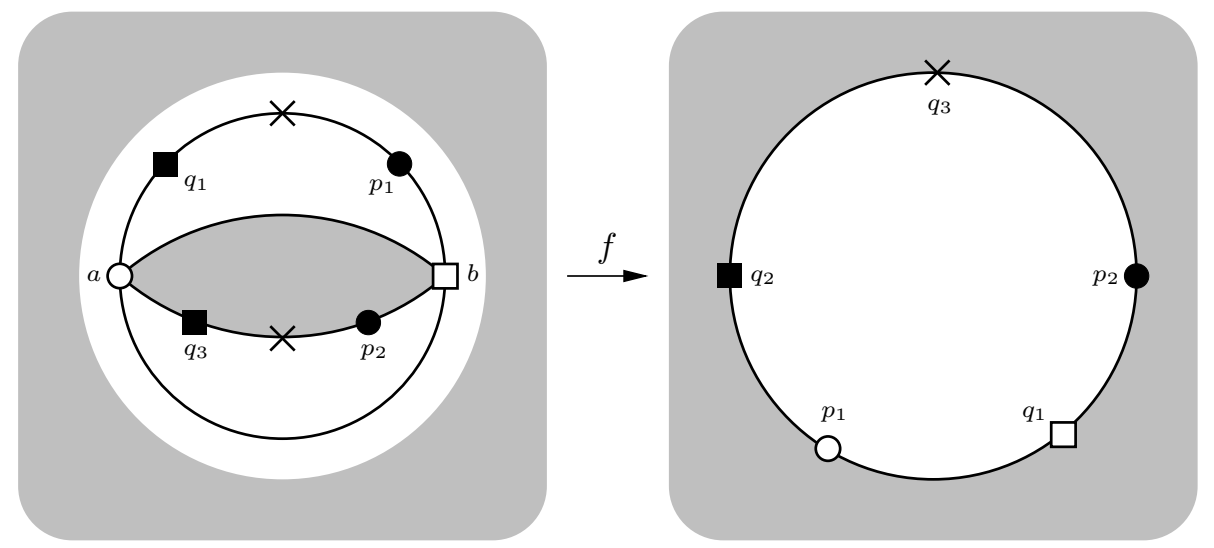

Figure 8. Case 3.

at $a$. Indeed the same $\operatorname{argument}$ always works when $p_{1}, p_{2}$ are adjacent on $\mathcal{C}$, i.e., Case 1 is impossible.

By exactly the same argument as above we can rule out that $q_{1}, q_{3}$ are adjacent on $\mathcal{C}$. Indeed as before they have to be in distinct white 1-tiles, since they are both preimages of $q_{2}$. If the white 1-tiles are connected either at $a$ or at $b$, there will be other postcritical points between $q_{1}, q_{3}$ when we traverse $\mathcal{C}^{1}$.

Case $2\left(p_{2}, q_{2}\right.$ are adjacent on $\left.\mathcal{C}\right)$.

We choose the orientation on $\mathcal{C}$ such that $q_{2}$ succeeds $p_{2}$ on $\mathcal{C}$. Consider the white 1-tile $X$ containing $p_{2}$ in its boundary $\partial X$. Then $p_{2}$ is succeeded on $\partial X$, hence on $\mathcal{C}^{1}$, by a 1 -vertex which is mapped to $q_{2}$ by $f$. This 1 -vertex must be $q_{1}$ or $q_{3}$. Hence on $\mathcal{C}^{1}$ the postcritical point $p_{2}$ is succeeded by either $q_{1}$ or $q_{3}$. Hence $\mathcal{C}^{1}$ is not obtained as a pseudo-isotopic deformation of $\mathcal{C}$ rel. post $(f)$ (in which case $p_{2}$ would be succeeded by $q_{2}$ on $\mathcal{C}^{1}$ ). Hence Case 2 does not happen.

From the above it follows that the cyclical order of the postcritical points on $\mathcal{C}$ is either $p_{1}, q_{1}, p_{2}, q_{3}, q_{2}, p_{1}$ or $p_{1}, q_{3}, p_{2}, q_{1}, q_{2}, p_{1}$. Here we are using that we can choose the orientation on $\mathcal{C}$ arbitrarily.

Case 3 (The cyclical order of the postcritical points on $\mathcal{C}$ is $p_{1}, q_{1}, p_{2}, q_{3}, q_{2}, p_{1}$ ).

The situation is illustrated in Figure 8. Since on $\mathcal{C}$ the point $p_{1}$ is succeeded by $q_{1}$ and $p_{2}$ is succeeded by $q_{3}$, the same has to hold on $\mathcal{C}^{1}$. Note however, that on $\mathcal{C}^{1}$ between $p_{1}, q_{1}$ and between $p_{2}, q_{3}$ there is a preimage of $q_{3}$. One of these preimages (marked with an $\mathrm{x}$ in the figure) of $q_{3}$ has to be $q_{2}$. Thus the cyclical ordering of the postcritical points on $\mathcal{C}^{1}$ does not agree with the one on $\mathcal{C}$.

Case 4 (The cyclical order of the postcritical points on $\mathcal{C}$ is $p_{1}, q_{3}, p_{2}, q_{1}, q_{2}, p_{1}$ ).

The situation is exactly analog to Case 3 . In the right of Figure 8 we have to interchange $q_{1}$ and $q_{3}$. Since $p_{1}$ is succeeded by $q_{3}$ and $p_{2}$ is succeeded by $q_{1}$ on $\mathcal{C}$, the same has to hold in $\mathcal{C}^{1}$. Thus in the right of Figure 8 we have to interchange $q_{1}$ and $q_{3}$ for the situation at hand. One of the points marked by $\mathrm{x}$ has to be $q_{2}$, which gives a contradiction as before. 


\section{OPEN QUESTIONS}

Let $f: \widehat{\mathbb{C}} \rightarrow \widehat{\mathbb{C}}$ be a rational map. Is the existence of an equator for $f$ sufficient for $f$ to arise as a mating? When $f$ is postcritically finite, this is widely expected, but there does not seem to be a proof in the literature.

A (pseudo-) equator $\mathcal{E}$ has to be orientation-preserving (pseudo-) isotopic to $f^{-1}(\mathcal{E})$. Orientation-reversing (pseudo-) equators seem to be as common as orientation-preserving ones. From such a pseudo-equator it is possible to construct a semi-conjugacy from $z^{-d}: S^{1} \rightarrow S^{1}$ to the map $f$ (here $d=\operatorname{deg} f$ ) (in the case when $f$ is postcritically finite and $\mathcal{J}(f)=\widehat{\mathbb{C}})$. There should be some sort of "orientationreversing mating" associated to such orientation-reversing (pseudo-) equators.

The form of Moore's theorem presented here allows one to shrink each rayequivalence class to a point by a pseudo-isotopy $H$. Is it possible to put further smoothness assumptions on $H$. In particular to embed $H$ in a holomorphic motion?

\section{REFERENCES}

[Bae27] R. Baer. Kurventypen auf Flächen. J. Reine Angew. Math., 156:231-246, 1927.

[Bae28] R. Baer. Isotopie von Kurven auf orientierbaren, geschlossen Flächen und ihr Zusammenhang mit der topologischen Deformation der Flächen. Reine Angew. Math., 159:101-116, 1928.

[Bea84] A. F. Beardon. A primer on Riemann surfaces, volume 78 of London Mathematical Society Lecture Note Series. Cambridge University Press, 1984.

[BFH92] Ben Bielefeld, Yuval Fisher, and John Hubbard. The classification of critically preperiodic polynomials as dynamical systems. J. Amer. Math. Soc., 5(4):721-762, 1992.

[BM11] M. Bonk and D. Meyer. Expanding Thurston Maps. Mathematical Surveys and Monographs. AMS, 2011. to appear.

[Dav86] R. J. Daverman. Decompositions of manifolds, volume 124 of Pure and Applied Mathematics. Academic Press Inc., Orlando, FL, 1986.

[DH93] A. Douady and J. H. Hubbard. A proof of Thurston's topological characterization of rational functions. Acta Math., 171(2):263-297, 1993.

[Dou83] A. Douady. Systèmes dynamiques holomorphes. In Bourbaki seminar, Vol. 1982/83, volume 105 of Astérisque, pages 39-63. Soc. Math. France, 1983.

[Eps66] D. B. A. Epstein. Curves on 2-manifolds and isotopies. Acta Math., 115:83-107, 1966.

[L.92] Tan L. Matings of quadratic polynomials. Ergodic Theory Dynam. Systems, 12(3):589620, 1992.

[Mey] D. Meyer. Expanding Thurston maps as quotients. Preprint.

[Mey11] D. Meyer. Invariant Peano curves of expanding Thurston maps. to appear in Acta Math., 2011.

[Mil99] John Milnor. Dynamics in one complex variable. Introductory lectures. Friedr. Vieweg \& Sohn, Braunschweig, 1999.

[Mil04] J. Milnor. Pasting together Julia sets: a worked out example of mating. Experiment. Math., 13(1):55-92, 2004.

[Mil06] J. Milnor. On Lattès maps. In Dynamics on the Riemann Sphere, A Bodil Branner Festschrift, Eur. Math. Soc. Hjorth and Petersen, 2006.

[Poi09] A. Poirier. Critical portraits for postcritically finite polynomials. Fund. Math., 203(2):107-163, 2009.

[Ree92] M. Rees. A partial description of parameter space of rational maps of degree two. I. Acta Math., 168(1-2):11-87, 1992.

[Shi00] M. Shishikura. On a theorem of M. Rees for matings of polynomials. In The Mandelbrot set, theme and variations, volume 274 of London Math. Soc. Lecture Note Ser., pages 289-305. Cambridge Univ. Press, Cambridge, 2000.

[SL00] M. Shishikura and Tan Lei. A family of cubic rational maps and matings of cubic polynomials. Experiment. Math., 9(1):29-53, 2000.

[Wit88] B. S. Wittner. On the bifurcation loci of rational maps of degree two. $\mathrm{PhD}$ thesis, Cornell University, 1988. 
Daniel Meyer, Jacobs University, School of Engineering and Science, Campus Ring 1, 28759 Bremen, Germany 